\title{
Massively parallelized molecular force manipulation with on demand thermal and optical control
}

Hanquan $\mathrm{Su}^{1,4}$ Joshua M. Brockman ${ }^{2,4}$ Yuxin Duan ${ }^{1,4}$ Navoneel Sen ${ }^{3}$ Hemani Chhabra $^{3}$ Alisina Bazrafshan ${ }^{1}$ Aaron T. Blanchard ${ }^{2}$ Travis Meyer $^{2}$ Brooke Andrews ${ }^{1}$ Jonathan P.K. Doye $^{3}$ Yonggang $\mathrm{Ke}^{1,2}$ R. Brian Dyer ${ }^{1}$ Khalid Salaita $^{1,2, *}$

${ }^{1}$ Department of Chemistry, Emory University, Atlanta, Georgia, 30322, United States. ${ }^{2}$ Wallace H. Coulter Department of Biomedical Engineering, Georgia Institute of Technology and Emory University, Atlanta, Georgia, 30322, United States. ${ }^{3}$ Physical and Theoretical Chemistry Laboratory, Department of Chemistry, University of Oxford, South Parks Road, Oxford OX1 3QZ, United Kingdom. ${ }^{4}$ These authors contributed equally to this work.

*Correspondence should be addressed to K.S. (k.salaita@emory.edu)

\section{This PDF file includes:}

Materials and Methods

Note S1-S3

Figure S1-S13

Table S1-S6 


\section{Materials and Methods}

$\underline{\text { Reagents }}$

All reagents were purchased from Sigma-Aldrich (St. Louis, MO) and were used as received, unless otherwise stated. N,N'-methylenebisacrylamide was purchased from Alfa Aesar (Haverhill, MA). Cy3B-NHS ester was purchased from GE healthcare Bio-Science (Pittsburgh, PA). Trishydroxypropyltriazolylmethylamine (THPTA) was purchased from Click Chemistry Tools (Scottsdale, AZ 85260). DMSO (99.5\%) and sodium bicarbonate (99.0\%) were acquired from EMD chemicals (Philadelphia, PA). P4-gel size exclusion beads were acquired from Biorad (Hercules, CA). Gold nanorods (AuNRs) were synthesized in-house and characterized by TEM. Based on TEM analysis, the mean diameter of AuNRs was $25 \times 100 \mathrm{~nm}$. Milli-Q water was obtained from a Nanopure system with $18.2 \mathrm{M}^{-1}-\mathrm{cm}$ resistivity. All oligonucleotides were purchased from Integrated DNA Technologies (Coralville, IA) and were purified either by reversephase HPLC or standard desalting.

\section{$\underline{\text { Synthesis of origami-polymer force clamp }}$}

First, cetyltrimethylammonium bromide (CTAB) stabilized AuNRs (size = $25 \times 100 \mathrm{~nm}$ ) were synthesized according to the protocol reported by Murray and colleagues ${ }^{1}$. After synthesis, the reaction mixture $(\sim 200 \mathrm{ml})$ was centrifuged at $5000 \mathrm{rpm}$ for $60 \mathrm{~min}$ and the supernatant was discarded. The AuNRs were resuspended in $90 \mathrm{ml}$ of DI water (concentration $=\sim 1 \mathrm{nM}$ ). CTAB was exchanged with N, N'-bis(acryloyl)cystamine (20 mg dissolved in $10 \mathrm{ml}$ ethanol) by vigorous stirring (700 rpm) for $12 \mathrm{~h}$. Next, $15 \mathrm{ml}$ Milli-Q water was heated to $70{ }^{\circ} \mathrm{C}$ with $\mathrm{N}_{2}$ purging in a three-neck flask. Next, $0.1 \mathrm{~g}$ of $\mathrm{N}$-isopropylmethacrylamide and $0.01 \mathrm{~g}$ of the crosslinking agent N,N'-methylenebisacrylamide were dissolved in $15 \mathrm{ml}$ Milli-Q water with vigorous stirring and continuously purged with $\mathrm{N}_{2}$ flow. Next, $7 \mathrm{ml}$ of the AuNR solution (described above, $\sim 1 \mathrm{nM}$ ) were centrifuged at $6500 \mathrm{rpm}$ for $10 \mathrm{~min}$, the supernatant was removed and AuNRs were dispersed in $1 \mathrm{ml}$ Milli-Q water and added to the reaction flask. Within 2 min, polymerization was initiated with the addition of $80 \mu \mathrm{l}$ of $0.1 \mathrm{M}$ of the free radical initiator, 2,2'azobis(2methylpropionamidine) dihydrochloride (AAPH). The reaction proceeded for $2 \mathrm{~h}$ and the alkyne functional group was introduced via dropwise injection of $0.01 \mathrm{ml}$ of propargyl methacrylate monomer $(80 \mu \mathrm{mol})$. After $1 \mathrm{~h}$, the reaction was stopped, and the turbid pNIPMAm particle solution was cooled to room-temperature. The product was centrifuged and dispersed in Milli-Q water. This was repeated at least three times to ensure sufficient removal of unreacted monomer. The core-shell structure and mono-dispersity of pNIPMAm particles were verified by TEM and dynamic light scattering (DLS) analysis, respectively. The particle concentration was calculated using Beer Lambert law. As reported by Christopher J. Orendorff and Catherine J. Murphy ${ }^{2}$, the extinction coefficient of gold nanorods $(42 \times 12.3 \mathrm{~nm}, \lambda=785 \mathrm{~nm}) \varepsilon 785 \mathrm{~nm}=4.6 \times 10^{9} \mathrm{M}^{-1} \mathrm{~cm}^{-1}$. Because the size of AuNR in this work is $\sim$ twice that of the reported values in all dimensions (L $\mathrm{x} \mathrm{W} \times \mathrm{H}$ ), we approximated the extinction coefficient of $25 \times 100 \mathrm{~nm}$ AuNR to be $\varepsilon 785 \mathrm{~nm}=2^{3} \mathrm{x}$ $4.6 \times 10^{9} \mathrm{M}^{-1} \mathrm{~cm}^{-1}=3.7 \times 10^{10} \mathrm{M}^{-1} \mathrm{~cm}^{-1}$.

The pNIPMAm particles were then decorated with single-stranded DNA (ssDNA) to facilitate the particle-DNA origami assembly. Briefly, $25 \mu \mathrm{l}$ of pNIPMAm particles $(\sim 1.25 \mathrm{nM})$ and $25 \mu l$ of ssDNA $\left(b^{*}, 100 \mu \mathrm{M}\right)$ was mixed in a $1.5 \mathrm{ml}$ Eppendorf tube. Then, in a separate tube, $5 \mu 1$ of $\mathrm{CuSO}_{4}$ $(20 \mathrm{mM})$ was added to $10 \mu \mathrm{l}$ of THPTA $(50 \mathrm{mM})$ solution to form a blue-colored Cu-THPTA complex (ligand to copper ratio is 5:1). Finally, $5 \mu 1$ of $\mathrm{Cu}$-THPTA complex was reduced by adding $5 \mu \mathrm{l}$ of $100 \mathrm{mM}$ freshly prepared sodium ascorbate solution (blue color to clear) and the mixture was added to the first tube containing pNIPMAm particle and ssDNA. Note that the Cu-THPTA 
needs to be reduced by sodium ascorbate before mixing with particle and ssDNA. The reaction was left at RT for 1 hour and washed 5 times by centrifugation at $5000 \mathrm{rpm}$ for 6 mins.

The origami template was assembled from single-stranded scaffold p7560, which was prepared from M13 phage using a previously reported method ${ }^{3-4}$. A 16-helix bundle (16HB) rod was designed in caDNAno, based on a 4 x 4 square lattice cross-section. To synthesize $16 \mathrm{HB}$ structure and incorporate DNA hairpin to $16 \mathrm{HB}$, a 10-fold excess of staple strands (100 nM) and 15 -fold excess of DNA hairpins $(150 \mathrm{nM})$ were mixed with p7560 scaffold strand $(10 \mathrm{nM})$ in folding buffer $\left(5 \mathrm{mM}\right.$ Tris, $1 \mathrm{mM}$ EDTA, $10 \mathrm{mM} \mathrm{MgCl}_{2}$ with a total volume of $50 \mu \mathrm{L}$. The mixture was denatured at $85^{\circ} \mathrm{C}$ for $10 \mathrm{~min}$, followed by a slow anneal from $60{ }^{\circ} \mathrm{C}$ to $25^{\circ} \mathrm{C}$ over $18 \mathrm{hrs}(-$ $\left.2^{\circ} \mathrm{C} / \mathrm{hr}\right) .16 \mathrm{HB}$ were purified from excess staples using agarose gel electrophoresis $(0.67 \%)$ in $0.5 \mathrm{X}$ TBE+Mg buffer (45 mM Tris, $45 \mathrm{mM}$ Boric acid, $1 \mathrm{mM}$ EDTA, $10 \mathrm{mM} \mathrm{MgCl} 2$ ). PEG precipitation was used to purify $16 \mathrm{HB}$ from excess staples in large scale synthesis $(30 \mathrm{nM})$. Briefly, the $16 \mathrm{HB}$ solution was mixed with precipitation buffer (15\% PEG8000, 1x TE, 505mM NaCl, $\left.10 \mathrm{mM} \mathrm{MgCl}_{2}\right)$ at a 1:1 ratio. Samples were centrifuged at $\sim 16,000 \mathrm{~g}$ for 25 minutes at $4{ }^{\circ} \mathrm{C}$ (there should be a pellet after centrifugation). Then the supernatant was removed, and DNA origami was resuspended with folding buffer. 16HB structures were characterized by agarose gel electrophoresis (1.5\%) and negative stain TEM imaging (1\% uranyl formate).

Assembly of origami-polymer force clamp

$15 \mu \mathrm{l}$ of pNIPMAm particles $(\sim 0.3 \mathrm{nM})$ were mixed with $50 \mu \mathrm{l}$ of $16 \mathrm{HB}$ origami beams $(\sim 30$ $\mathrm{nM})$. The mixture was incubated overnight at room temperature. The solution was then centrifuged at least 5 times at $5000 \mathrm{rpm}$ for 6 mins. Finally, the sample was re-dispersed in $\sim 40 \mu \mathrm{l}$ buffer B ( 5 $\mathrm{mM}$ Tris- $\mathrm{HCl}, 10 \mathrm{mM} \mathrm{MgCl}_{2}$ and $1 \mathrm{mM}$ EDTA at $\left.\mathrm{pH}=8\right)$. This translates to $\sim 10^{9} \mathrm{OPFCs}(0.3 \mathrm{nM}$ $* 15 \mu \mathrm{l} * \mathrm{~N}_{\mathrm{A}}$ ), $\sim 10^{11}$ origami beams ( 200 origami structures on each particle), and $\sim 10^{12}$ target molecules.

Transmission electron microscopy (TEM)

TEM measurements were acquired on a Hitachi H-7500 transmission electron microscope at an accelerating voltage of $80 \mathrm{kV}$ in the Robert P. Apkarian Integrated Electron Microscopy Core at Emory University. A $5 \mu 1$ sample (AuNRs or OPFCs) was deposited onto a 200-mesh carbon coated copper grid (Electron Microscopy Sciences). After $60 \mathrm{sec}$ of incubation, excess liquid was wicked away. This incubation step was repeated three times. OPFCs sample preparation, negative staining was used after the sample incubation step described above. The specimens were stained by adding $5 \mu \mathrm{l}$ of $1 \%$ uranyl formate solution onto the TEM grid. After $60 \mathrm{sec}$ incubation, the excess liquid was wicked away. The sample grids were subsequently dried and stored in a desiccator.

\section{DNA labeling}

A mixture of oligonucleotide $(0.01 \mathrm{ml}, 10 \mathrm{nmol})$ and excess Cy3B-NHS ester $(0.05 \mathrm{mg}$ dissolved in $0.01 \mathrm{ml}$ DMSO $)$ in $0.1 \mathrm{M}$ sodium bicarbonate solution $(0.08 \mathrm{ml})$ was reacted at roomtemperature for $12 \mathrm{~h}$. The product was subjected to P4-gel filtration to desalt and to remove salts and unreacted dye. Reverse phase HPLC (solvent A: 0.1 M TEAA, solvent B: 100\% MeCN; initial condition was $10 \% \mathrm{~B}$ with a gradient of $1 \% / \mathrm{min}$, flow rate: $1 \mathrm{ml} / \mathrm{min}$ ) was applied to further purify the product. Reaction products were purified using a L10NM8 column (diameter: $4.6 \mathrm{~mm}$; length: $250 \mathrm{~mm}$ ) in a reverse phase binary pump HPLC that was coupled to a diode array detector (Agilent 1100). 
Equilibrium fluorescence measurements

Fluorescence spectra of Cy3B labeled DNA hairpin were obtained on the Dual-Fl Fluorometer (Horiba Scientific, Edison, New Jersey) with a $520 \mathrm{~nm}$ excitation. The samples were prepared at a concentration of $150 \mathrm{nM}$. Temperature dependent spectra were obtained from $20^{\circ} \mathrm{C}$ to $90{ }^{\circ} \mathrm{C}$ in increments of $2.5^{\circ} \mathrm{C}$ using a peltier temperature controller. The solution temperature was held for 2 mins before collecting steady-state fluorescence spectra of the hairpins at each temperature. The $\mathrm{Cy} 3 \mathrm{~B}$ fluorescence emission peak was acquired at $575 \mathrm{~nm}$. Integration time $=5$ sec, detector accumulation $=3$.

\section{Time-resolved T-jump fluorescence spectroscopy}

Temperature-jump fluorescence relaxation experiments were performed on a custom-built instrument as previously described $(25,51)$. Briefly, a Q-switched Ho:YAG laser (AQS-Ho-YAG, IPG Phototonics Corp., Oxford, MA) was used to generate a temperature jump of approximately $8-10^{\circ} \mathrm{C}$. Temperature jumps are generated from the $\mathrm{H}_{2} \mathrm{O}$ absorbance bands in the far IR. Fluorescence emission was probed using $490 \mathrm{~nm}$ excitation generated by frequency-doubled Ti:Sapphire $(980 \mathrm{~nm})$ pumped by a Chameleon laser (Coherent, Santa Clara, CA). Sample emission fluorescence was collected at $\sim 90^{\circ}$ to the $\mathrm{CaF}_{2}$ chamber and focused through a bandpass filter $(514 \mathrm{~nm}-570 \mathrm{~nm})$. The emission was measured using a Hamamatsu R7518 photomultiplier tube (Hamamatsu Photonics K. K., Hamamatsu, Japan). The signal was digitized and averaged (3000 shots) by a using a Wavesurfer 62Xs-B oscilloscope (Teledyne LeCroy, Chestnut Ridge, NY).

\section{Data acquisition}

Temperature jump experiments were performed on the origami-polymer force clamp or the DNA hairpin samples in buffer B (5 mM Tris- $\mathrm{HCl}, 10 \mathrm{mM} \mathrm{MgCl} 2$ and $1 \mathrm{mM}$ EDTA $)$ at $\mathrm{pH}=8$. This buffer has been reported for preserving the intact structure of DNA origami (52). Rhodamine B solution $(150 \mathrm{nM})$ was used as a reference to determine the temperature jump. The temperature dependency of Rhodamine B fluorescence was calibrated on the temperature-jump setup with a $490 \mathrm{~nm}$ excitation. The temperature was adjusted via a water bath and held for 5 mins. Then, Rhodamine B fluorescence signal was recorded (using 3000 shots) and measured using the same instrumental setup and filters as those used for the T-jump and Force-jump measurements.

\section{Note S1. Geometric analysis of DNA origami design parameters.}

We used geometric calculations and chemo-mechanical modeling to assess the subset of the rod length and thickness parameter space that can conceivably result in particle collapse-drive tension probe opening. Specifically, we tested for length-thickness pairs that satisfied four conditions.

1. Two closed tension probes (positioned $15 \mathrm{~nm}$ from each end of the rod) need to be able to simultaneously bind to the relaxed particle. To test for this condition, we used the Petrosyan approximation of the worm-like-chain model ${ }^{5}$ to calculate the closed tension probe's forceextension curve. We used this force-extension curve to find that $F=4.7 p N$ (which is equal to the probe's $F_{1 / 2}{ }^{6}$ ) when the closed tension probe's end-to-end extension is $17.3 \mathrm{~nm}$. If the origami rod and the particle are treated as rigid and non-intersecting, then the length at which tethers can only 
connect to the particle with a force $\geq 4.7 p N$ ( $L_{\text {cutoff }}$ ) can be calculated by re-arranging the equation:

$$
17.3 \mathrm{~nm}=\sqrt{(250 \mathrm{~nm})^{2}+\left(\frac{L_{\max }}{2}-(15 \mathrm{~nm})\right)^{2}}-(250 \mathrm{~nm})
$$

where $250 \mathrm{~nm}$ is the particle's radius. This yielded $L_{\max }=219 \mathrm{~nm}$. This condition is satisfied only when $L \leq L_{\max }$. This result would change with the particle's diameter or the length of the closed tension probe.

2. Closed tension probes need to be opened by particle collapse. To test for this condition, we tested all length-thickness pairs where the above condition was satisfied. Then, we checked to make sure that particle collapse (which results in a decrease of particle radius from $250 \mathrm{~nm}$ to 125 $\mathrm{nm}$ ) could transfer the tension probe from a closed state (tether extension $<17.3 \mathrm{~nm}$ ) to an opened state (tether extension $>17.3 \mathrm{~nm}$ ). We found that when $L \leq 65 \mathrm{~nm}$, particle collapse never caused tension probes to transition from a closed state to an open state. The regions of the length-thickness parameter space that fail to meet this or the previous condition are shown in green below.

3. Particle collapse should not result in "buckling" of the DNA origami rod. To test for this condition, we measured the critical buckling force $\left(F_{c}\right)$ using an equation presented by Liedl et. al 7:

$$
F_{c}=\pi^{2} P k_{B} T / L^{2}
$$

where $P$ is the persistence length of the bundle and $k_{B} T=4.114 \mathrm{pN} \mathrm{nm}$ is the Boltzmann's constant at room temperature. Based on a previous model presented by Castro et. $a l^{8}$, we estimate $P$ as $P=(53 \mathrm{~nm})\left(N_{\text {duplex }}\right)^{1.94}$ where $N_{\text {duplex }}$ is the number of duplexes in the bundle. The region of the length-thickness parameter space that fails to meet this condition (e.g. $F_{c} \leq 4.7 p N$ ) is shown in red below.

The above analysis also does not take into account that what's important for the bending of the origami is not simply the force being applied by a single hairpin, but the effective net force due to all the hairpins and linkers.

4. The rod should have dimensions that are appropriate for self-assembly. To test for this condition, we checked if the number of nucleotides in the rod, which we estimated as $L N_{\text {duplex }} /(0.34 \mathrm{~nm})$, was lower than 7,560 nt (the length of the scaffold used in this work). The region of the lengththickness parameter space that fails to meet this condition (e.g. $F_{c} \leq 4.7 p N$ ) is shown with grey stripes above

We found that our selected condition $\left(N_{\text {duplex }}=16\right.$ and $\left.L=130 \mathrm{~nm}\right)$, denoted with a black dot in the figure below, satisfied all four of this conditions.

For the subset of the parameter space in which all four conditions were satisfied, we also estimated the fraction of tension probes that would be opened by particle collapse. To do this, we used Boltzmann distribution calculations to estimate the distribution of particle attachment points and then used the calculated Boltzmann distribution to estimate the percentage of tension probes that transition from a closed state to an opened state following particle collapse. We found that the percentage increased from $\sim 0 \%$ to $\sim 100 \%$ from $L \approx 65 \mathrm{~nm}$ to $L \approx 125 \mathrm{~nm}$, as shown via color 
within the figure below. These calculations were performed by assuming that the particle acts as a rigid sphere that decreases in size during collapse and that the rod is rigid as well. In reality, both structures likely undergo relaxation in response to force transmitted through the tension probes that will reduce the force experienced by the tension probes (and, by extension, reduce the fraction of tension probes that are opened by collapse). The OxDNA calculations further elaborate on this point.

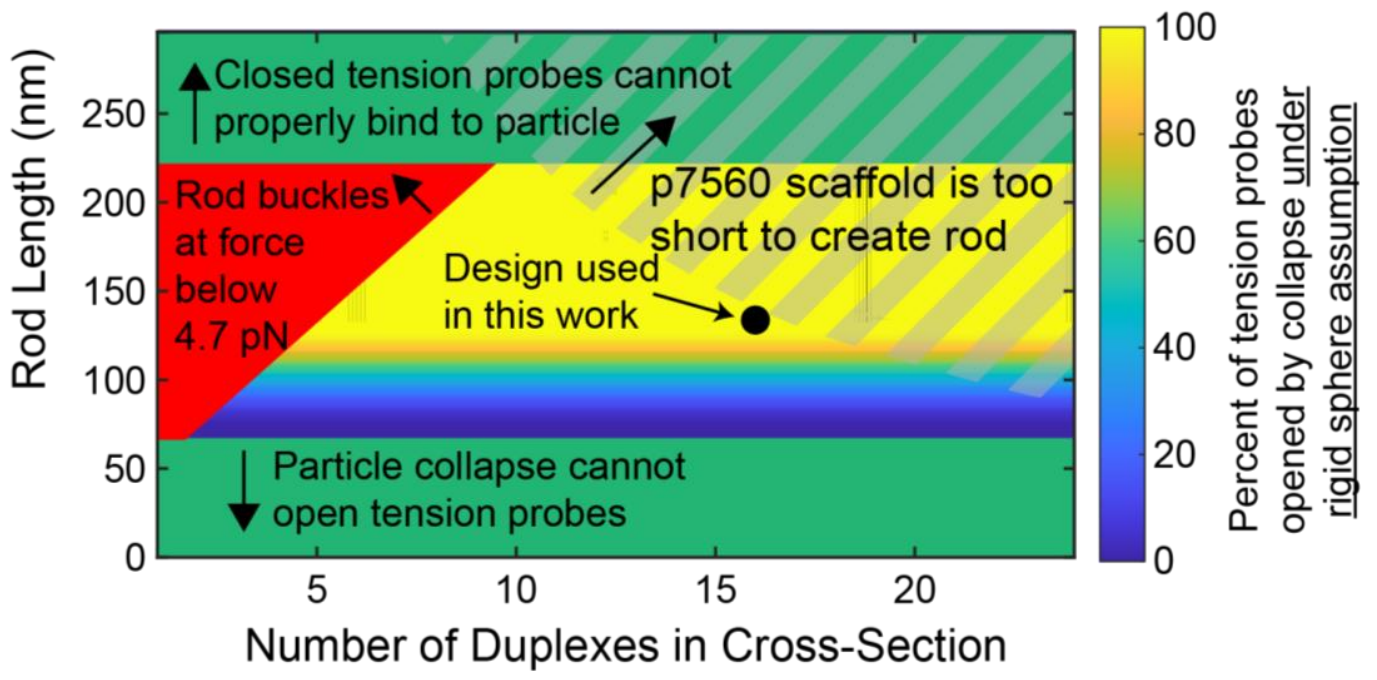

\section{Note S2. Force calculation}

Here, we considered the DNA hairpin as a classic two-state system, where a hairpin structure transformed between folded and unfolded state, unfolding rate constant $k_{\mathrm{u}}$ and $k_{\mathrm{f}}$, respectively. Briefly, calculation of the force applied by OPFC can be described in the following steps:

1. Steady-state fluorescence measurement to determine the $\mathrm{K}_{\mathrm{eq}}$

The equilibrium reaction constant $\mathrm{K}_{\mathrm{eq}}$ can be measured by a steady-state temperature dependent fluorescence measurement of the DNA hairpin structure or a molecular beacon structure which contain the identical region of DNA hairpin stem-loop. At each temperature, $\mathrm{K}_{\mathrm{eq}}$ of DNA hairpin stem melting is expressed as equation below, where $f$ is the fraction of hairpins in the open state:

$$
K=\frac{[\text { open }]}{[\text { closed }]}=\frac{k_{u}(0 p N, T)}{k_{f}(0 p N, T)}=\frac{f}{1-f}
$$

2: Pump-probe temperature-jump measurement to determine the $k_{r}(0 \mathrm{pN}, \mathrm{T})$

The T-jump is a relaxation method where a shift in temperature acts as a perturbation. A transient temperature pump is applied and shifts the equilibrium position of the system. Then, then the dynamic change of the system can be detected by a time-resolved probe beam. Specifically, in our pump-probe setup, the time-resolved fluorescence signal was collected by a PMT and fitted to a single-exponential function. From the fitting, the relaxation lifetime (which is equal to $\left.1 / k_{r}\right) \tau_{\mathrm{r}}(0$ $\mathrm{pN}, \mathrm{T})$ at each temperature was extracted. The relaxation rate following the perturbation $\left(k_{r}(0 \mathrm{pN}\right.$, $\left.\mathrm{T})=1 / \tau_{\mathrm{r}}(0 \mathrm{pN}, \mathrm{T})\right)$ is defined as the sum of the unfolding rate $k_{u}(0 \mathrm{pN}, \mathrm{T})$ and the re-folding rate $k_{f}$ $(0 \mathrm{pN}, \mathrm{T})$ :

$$
\mathrm{k}_{\mathrm{r}}(0 \mathrm{pN}, \mathrm{T})=1 / \tau_{\mathrm{r}}(0 \mathrm{pN}, \mathrm{T})=k_{u}(0 \mathrm{pN}, \mathrm{T})+k_{f}(0 \mathrm{pN}, \mathrm{T})
$$


3:Calculation of $k_{u}(0 \mathrm{pN}, \mathrm{T})$ and $k_{f}(0 \mathrm{pN}, \mathrm{T})$

From steps $1 \& 2$, the $k_{u}(0 \mathrm{pN}, \mathrm{T})$ and $k_{f}(0 \mathrm{pN}, \mathrm{T})$ were calculated and expressed as:

$$
\begin{aligned}
& k_{u}(0 \mathrm{pN}, \mathrm{T})=\frac{1}{\tau_{\mathrm{r}}(0 \mathrm{pN}, \mathrm{T})} \times \frac{K_{e q}}{1+K_{e q}} \\
& k_{f}(0 \mathrm{pN}, \mathrm{T})=\frac{1}{\tau_{\mathrm{r}}(0 \mathrm{pN}, \mathrm{T})} \times \frac{1}{1+K_{e q}}
\end{aligned}
$$

4. Calculation of rate constants $k_{u}(0 \mathrm{pN}, \mathrm{T})$ and $k_{f}(0 \mathrm{pN}, \mathrm{T})$ at low temperature $\left(\mathrm{T}_{\text {final }}<45^{\circ} \mathrm{C}\right)$

Because the time-resolved $\mathrm{T}$-jump with $\mathrm{T}_{\text {final }}<45^{\circ} \mathrm{C}$ did not show significant fluorescence signal across the measured timescale, it is not possible to directly measure $k_{r}$ or calculate the $k_{u}(0 \mathrm{pN}, \mathrm{T})$ and $k_{f}(0 \mathrm{pN}, \mathrm{T})$ at these temperatures. In order to quantify the $k_{u}$ and $k_{f}$, at these temperatures we constructed an Arrhenius plot with the unfolding rate $k_{u}$ at high temperature $\left(\mathrm{T}_{\text {final }} \geqslant 45^{\circ} \mathrm{C}\right)$ and extrapolated it to the low temperature regime $\left(\mathrm{T}_{\text {final }}<45^{\circ} \mathrm{C}\right)$ as shown in Figure 3e. The Arrhenius plot was fitted by a linear fitting function: $\ln \left(k_{u}(0 \mathrm{pN}, \mathrm{T})\right)=100.07-30242.57 *(1 / \mathrm{T})$. From the extrapolation, $\ln \left(k_{u}\left(0 \mathrm{pN}, 45^{\circ} \mathrm{C}\right)\right)=5.017$ and $k_{u}\left(0 \mathrm{pN}, 45^{\circ} \mathrm{C}\right)=151 \mathrm{~s}^{-1}$. To validate this fitting and the unfolding rate from the extrapolation, we compared the $k_{u}(0 \mathrm{pN})$ to the reported values at $23^{\circ} \mathrm{C}$ for similar structure from Woodside and colleagues ${ }^{9}$. Importantly, because the $k_{u}(0 \mathrm{pN})$ is temperature dependent, we further extrapolate the Arrhenius plot to $23^{\circ} \mathrm{C}$ and get $k_{u}\left(0 \mathrm{pN}, 23^{\circ} \mathrm{C}\right)$ $=\exp (-2.04)=0.13 \mathrm{~s}^{-1}$. This number agrees with the reported value $k_{u}\left(0 \mathrm{pN}, 23^{\circ} \mathrm{C}\right)=\exp (-4.1)=$ $0.017 \mathrm{~s}^{-1}$ from a similar DNA structure (10R50/T4) with 10-mer stem with 50\% GC content and 4-nt loop (GAGTCTCCTA-TTTT-TAGGAGACTC). Note that the unfolding rate of our DNA structure is greater than the reported values for other stem loop hairpins. This is likely due to the low GC content $(\sim 22 \%)$ in our stem, different experimental conditions ( $200 \mathrm{mM}$ monovalent salt in Woodside's paper and $5 \mathrm{mM}$ Tris- $\mathrm{HCl}, 10 \mathrm{mM} \mathrm{MgCl} 2$ and $1 \mathrm{mM}$ EDTA in our work), and the shorter loop (7 in our work compared to 4 nt loop used by Woodside et al.). Accordingly, the folding rate $k_{f}(0 \mathrm{pN})$ at low temperature can be calculated using the $\mathrm{K}_{\mathrm{eq}}$ from the steady-state measurement in step 1: $k_{f}(0 \mathrm{pN}, \mathrm{T})=\frac{K_{e q}}{k_{u}(0 \mathrm{pN}, \mathrm{T})}$

\section{Pump-probe force-jump measurement to determine the $k_{r}(\mathrm{~F}, \mathrm{~T})$}

Similar to the T-jump measurement for the DNA hairpin, the relaxation lifetime $\tau_{\mathrm{r}}(\mathrm{F}, \mathrm{T})$ was acquired from single exponential fitting of the time-resolved fluorescence signal. The relaxation rate under force $k_{r}(\mathrm{~F}, \mathrm{~T})=1 / \tau_{\mathrm{r}}(\mathrm{F}, \mathrm{T})$, is defined as the sum of the unfolding rate $k_{u}(\mathrm{~F}, \mathrm{~T})$ and folding rate $k_{f}(\mathrm{~F}, \mathrm{~T})$ with the application of force.

$$
\mathrm{k}_{\mathrm{r}}(\mathrm{F}, \mathrm{T})=1 / \tau_{\mathrm{r}}(\mathrm{F}, \mathrm{T})=k_{u}(\mathrm{~F}, \mathrm{~T})+k_{f}(\mathrm{~F}, \mathrm{~T})
$$

6. Estimation of the force

We next calculated the force using the Bell model which was first developed by G. Bell in 1978 ${ }^{10}$. The Bell model predicts how forces modulate the kinetics of an idealized two-state system separated by a single barrier under constant force. In the Bell model, the force effectively modulates the $k_{u}$ and $k_{f}$ as follows ${ }^{10}$ : 


$$
\begin{aligned}
& k_{u}(F, T)=k_{u}(0 p N, \mathrm{~T}) \times \exp \left(\frac{F \Delta x_{u}^{\ddagger}}{\mathrm{k}_{\mathrm{B}} \mathrm{T}}\right) \\
& k_{f}(F, \mathrm{~T})=k_{f}(0 p N, \mathrm{~T}) \times \exp \left(\frac{-F \Delta x_{f}^{\ddagger}}{\mathrm{k}_{\mathrm{B}} \mathrm{T}}\right)
\end{aligned}
$$

$\Delta \mathrm{x}_{\mathrm{u}}^{\ddagger}$ and $\Delta \mathrm{x}_{\mathrm{f}}^{\ddagger}$ are the distance to the transition state. Previously, Woodside's work reported experimental measurement and theoretical estimation of $\Delta \mathrm{x}_{\mathrm{u}}{ }^{*}$ of the similar DNA hairpin sequence $\left(\Delta \mathrm{xu}_{\mathrm{u}}^{\ddagger}=4.0 \mathrm{~nm}, \Delta \mathrm{x}_{\mathrm{f}}^{\ddagger}=5.1 \mathrm{~nm}, 10 \mathrm{R} 50 / \mathrm{T} 4\right)^{9}$.

From step 5, the relaxation time of force-jump experiment can now be written as:

$$
k_{r}(\mathrm{~F}, \mathrm{~T})=\frac{1}{\tau_{r}(\mathrm{~F}, \mathrm{~T})}=k_{u}(0 p N, \mathrm{~T}) \times \exp \left(\frac{F \Delta x_{u}^{\ddagger}}{\mathrm{k}_{\mathrm{B}} \mathrm{T}}\right)+k_{f}(0 p N, \mathrm{~T}) \times \exp \left(\frac{-F \Delta x_{f}^{\ddagger}}{\mathrm{k}_{\mathrm{B}} \mathrm{T}}\right)
$$

Therefore, the only unknown variable, force $(\mathrm{F})$ can be calculated. At $45^{\circ} \mathrm{C}$, we calculated the effective force generated by the OPFC is $3.4 \pm 0.45 \mathrm{pN}$ from triplicate measurements of the Fjump and T-jump experiments (Figure S11\&12).

Note S3. OxDNA simulation

\section{OxDNA Model}

(a)
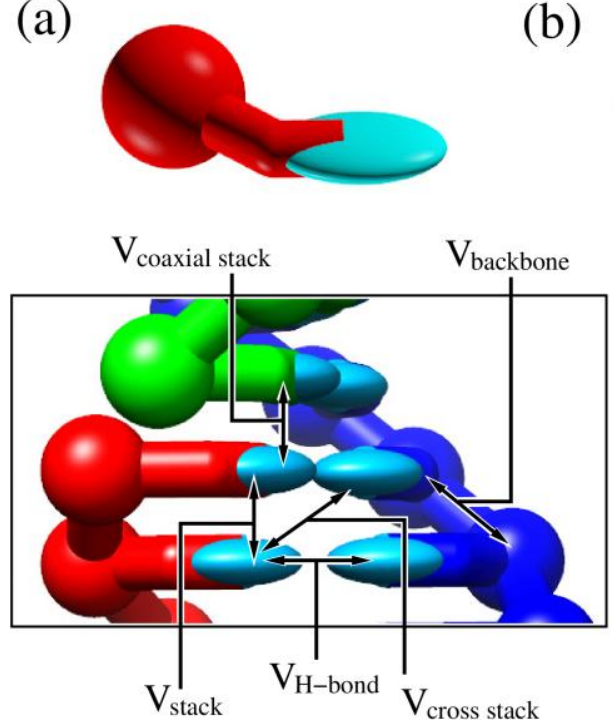

(b)
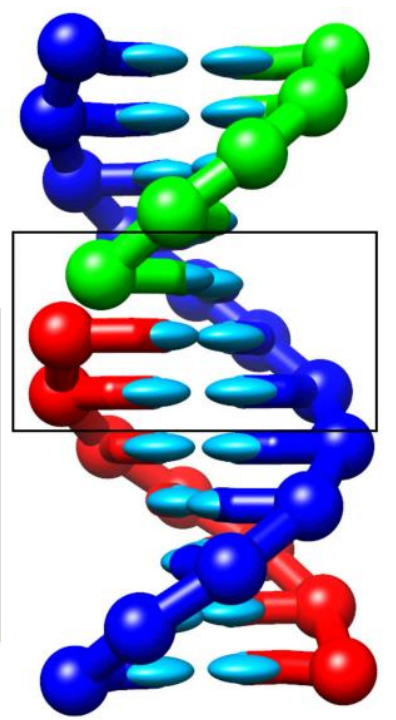

Note Figure S1: (a) A representation of a rigid oxDNA nucleotide. (b) A 12-base-pair DNA double helix as represented by oxDNA. The inset shows the different types of interactions that are accounted for in the oxDNA model, namely, hydrogen-bonding between complementary bases, (coaxial) stacking interactions between bases, cross-stacking interactions between diagonally-opposite bases in a double helix, and a FENE potential between backbone sites, as well as excluded-volume repulsions associated with the backbone and base sites.

OxDNA is a coarse-grained model simulation of DNA at the nucleotide level ${ }^{11-13}$. Note Figure S1 shows a double helix as represented by oxDNA with the interactions that contribute to the model illustrated. The parameters of the model have been fitted to reproduce the structure, thermodynamics and mechanics of double-stranded and single-stranded DNA with a particular focus on the thermodynamics of hybridization. We use the second-generation version of the oxDNA model (sometimes called “oxDNA2") introduced by Snodin et al. ${ }^{13}$; this included changes 
that improved the description of large DNA nanostructures such as origamis. OxDNA's excellent reproduction of the structure of DNA origamis has been further confirmed by a detailed comparison to a high-resolution origami structure obtained by cryoEM ${ }^{14-15}$. Consequently, it has been widely used to model the structure of DNA origamis with much success. Particularly relevant to the current application is oxDNA's ability to describe the mechanical properties of origamis, because of the tensile forces applied by the linkers and hairpins to the origami force clamp. Importantly, it has been shown to provide a reasonable description of both the elastic moduli of DNA origamis ${ }^{16}$ and the local unravelling of DNA origami through unbinding of staple domains due to applied tension ${ }^{17}$. Note, that the tensile forces were found to be not sufficiently large to cause any staple unbinding in the case of the OPFC. Also important to the current application is oxDNA's ability to capture the effects of tension on small DNA motifs, such as hairpins and duplexes ${ }^{18-19}$. This stems from its good description of the thermodynamics of hybridization and hairpin formation ${ }^{11}$, the force-extension curves of single-stranded DNA ${ }^{12}$ and the persistence length of double-stranded DNA ${ }^{11,13}$. For all these reasons oxDNA is particularly well-suited to describe the OPFC. Similarly, it has also been used to calibrate the origami force sensor ${ }^{20}$ of Nickels et al. ${ }^{21}$.

\section{System setup}

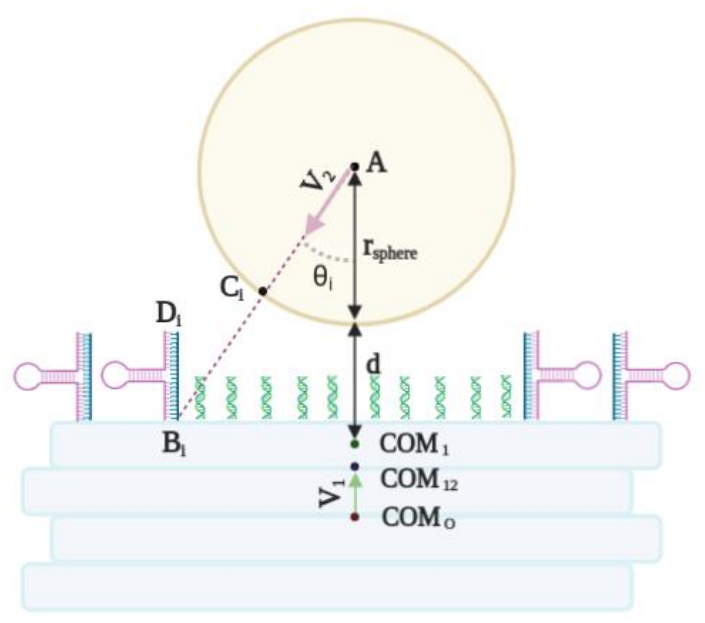

Note Figure S2: Schematic to show how the parameters for modelling the polymer sphere in the oxDNA simulations are chosen (for clarity only two of the three hairpins on each side are shown). The relative sizes of the objects are not to scale.

In the oxDNA simulations we do not model the sphere explicitly, but instead model it effectively by a set of harmonic traps that constrain the positions of the end nucleotides that are attached to the polymer sphere. These traps lie on the surface of a sphere with a radius appropriate for the given temperature. Note Figure S2 illustrates our approach for calculating the positions of the traps at low temperature. First, we calculate the centers of mass of the origami (COMo), its top layer $\left(\mathbf{C O M}_{1}\right)$ and its two top-most layers $\left(\mathbf{C O M}_{12}\right)$. From the center of the top layer, we then added a vector of magnitude $\left(d+r_{\text {sphere }}\right)$ in the direction given by the vector $\mathbf{V}_{\mathbf{1}}=\mathbf{C O M}_{\mathbf{1 2}}$ - COMo to get the center of the sphere (A). We assume that the hairpins/linkers are attached to the closest point on the polymer sphere. The details are as follows. For each linker/hairpin, we define their point of attachment $\left(\mathbf{B}_{\mathbf{i}}\right)$ to the origami based on an equilibrated origami configuration; we then 
define the vector $\mathbf{V}_{\mathbf{2}}=\mathbf{B}_{\mathbf{i}}-\mathbf{A}$ for each linker/hairpin. In the direction of $\mathbf{V}_{\mathbf{2}}$ we add a vector of magnitude $r_{\text {sphere }}$ to $\mathbf{A}$ to get $\mathbf{C}_{\mathbf{i}}$ the trap center for that particular linker/hairpin that represents its attachment to the sphere. To calculate the trap positions for the sphere $\mathbf{C}_{\mathbf{i}}$ at high temperature, we assumed that the sphere underwent a uniform contraction, keeping the angle $\left(\theta_{i}\right)$ the same but decreasing $r_{\text {sphere }}$ from $280 \mathrm{~nm}$ at $313 \mathrm{~K}$ to $205 \mathrm{~nm}$ at $328 \mathrm{~K}$. After all the trap centers are calculated for the linkers and hairpins, the origami is simulated at $313 \mathrm{~K}$ and $328 \mathrm{~K}$ until the hairpins have reached the required distance from the center of the sphere and the forces have equilibrated.

\section{Simulation details}

L110nm is our initial model system, with the six hairpins located at the two extremes of the origami in groups of 3 . To investigate the effect of the position of the hairpins on the origami on the force exerted by the sphere, we simulated five more systems (L90nm - L10nm) where the hairpins were progressively moved to the center of the origami. L10nm had all the hairpins concentrated at the center of the origami. The $\mathbf{\Delta 3 2}$ system had the hairpins in the same position as $\mathbf{L} \mathbf{1 1 0 n m}$ but lacked multiple staple strands at the center of the origami. The absence of staple strands makes $\Delta \mathbf{3 2}$ much more flexible than the other origamis. We assume the sphere to have a radius of 280 $\mathrm{nm}$ at $313 \mathrm{~K}$ and $205 \mathrm{~nm}$ at $328 \mathrm{~K}$. We perform MD simulations to simulate each system at $313 \mathrm{~K}$ and $328 \mathrm{~K}$ in the canonical $N V T$ ensemble 22 ; we will refer to these as the low and high temperature states. The coupling between the system and a heat bath is emulated by employing an Andersenlike thermostat ${ }^{23}$. We chose harmonic traps with a force constant of $28.545 \mathrm{pN} / \mathrm{nm}$ to model the effect of the sphere and run our simulations until the forces have equilibrated. The only difference between the high and the low temperature states is the radius of the polymer sphere. At low temperature, the trap positions on the sphere are closer to the respective hairpins and linkers, and consequently the force felt is low. At high temperature, the sphere shrinks, and the trap positions all move towards the center of the sphere thereby increasing the distance between them and the hairpins and linkers which results in larger forces. The forces experienced by the origami at high temperature has a compressive component as well as a lateral bending component because of the sphere shrinkage. We also perform simulations at high temperature with "mutual traps" between the nucleotides in the hairpin so that the hairpins are prevented from unfolding, thus allowing us to obtain the forces experienced in the absence of any hairpin unravelling.

\section{Assumptions}

There are a number of assumptions in the setup of the simulations of which it is important to be aware. First, we assume that when the hairpins and linkers attach to the polymer sphere (in the low temperature regime for the sphere size) they attach to the closest point on the polymer spheres, which we assume are perfectly smooth. In practice, although this is a reasonable assumption for the average position of the attachment points across the ensemble of origamis, we expect there to be a distribution of such points across the ensemble of origamis. We do not account for this potential heterogeneity in population of the OPFCs. Second, we do not explicitly account for the elastic mechanical properties of the sphere. For example, the spring constant for our harmonic traps is in the "stiff-sphere" limit where the obtained forces do not depend on the spring constant because the origami is more compliant than the traps. Third, we assume that all the hairpins and linkers are simultaneously attached to the sphere. The parallel application of the forces through all these linkers and hairpins is the reason for the significant bending of the origami. If not all linkers/harpins are bound, then the total applied force on the origami is likely to be less and the 
origami less bent, which in turn is likely to lead to higher forces through the hairpins/linkers that are attached.

We also note that upon close inspection that the 16HB origami has an inherent twist of around $-80^{\circ}$ between the two ends. Such twist is expected for an origami based on a square lattice of helices without insertions or deletions ${ }^{24}$. However, this twist is too low to have significantly affect the geometric assumptions of the design.

\section{Further results.}

\begin{tabular}{|c|c|c|}
\hline System & $\mathrm{T}=313 \mathrm{~K}$ & $T=328 \mathrm{~K}$ \\
\hline $\mathrm{L}_{10 \mathrm{~nm}}$ & & \\
\hline $\mathrm{L}_{30 \mathrm{~nm}}$ & & \\
\hline $\mathrm{L}_{50 \mathrm{~nm}}$ & $x^{2} h+1, y$ & \\
\hline $\mathrm{L}_{70 \mathrm{~nm}}$ & 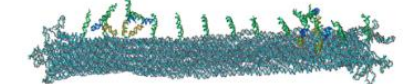 & \\
\hline $\mathrm{L}_{90 \mathrm{~nm}}$ & 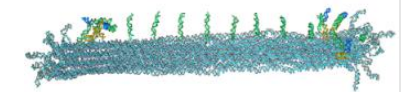 & \\
\hline $\mathrm{L}_{110 \mathrm{~nm}}$ & & \\
\hline $\mathrm{L}_{110 \mathrm{~nm}}(\Delta 32)$ & & \\
\hline
\end{tabular}

Note Figure S3: Snapshots of C1-C6 and $\Delta 32$ at low and high temperatures. At high

temperature, a strong bend is visible in all C1-C6 while $\Delta 32$ is kinked.

Configurations for each of the origami force clamps are illustrated in Note Figure S3 at both low and high temperature. The combined effect of the forces on the hairpins and linkers causes the origami to bend significantly. Although, the force acting through each individual hairpin/linker is not particularly high, the sum of all such forces is substantial. At low temperature, we note that all the hairpins and linkers are roughly oriented radially towards the center of the sphere, meaning they are co-linear with the radius vector at that point on the sphere. We note from the average forces that as the sphere shrinks, the hairpins and linkers in the center of the origami tend to be in a state of compression rather than tension because of their proximity to the surface of the sphere. At high temperature, the origami bends more as a result of the higher average force

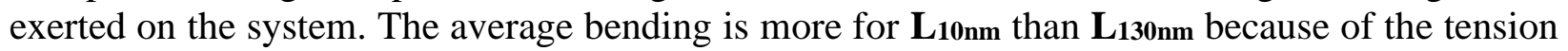
in the outer linkers in $\mathbf{L} \mathbf{1 0} \mathbf{n m}$ whereas in $\mathbf{L} \mathbf{1 3 0} \mathbf{n m}$, the hairpins can also absorb the stress by unfolding. The deletion of 16 staple strands in $\mathbf{\Delta 3 2}$ gives the origami significantly more flexibility and therefore it bends more at high temperature. We observe that the average angle of bending between the two origami blocks either side of the flexible section is significantly larger at $328 \mathrm{~K}\left(42.616^{\circ}\right)$ compared to at $313 \mathrm{~K}\left(13.066^{\circ}\right)$ because the origami has deformed to absorb much of the strain due to the particle collapse, thereby making hairpin unfolding much less likely (Table S1). 
Table S1: Average angle of origami bending and standard error along hairpins.

\begin{tabular}{|c|c|c|c|c|c|c|}
\hline Configuration & $\begin{array}{c}\text { Angle of bending } \\
\text { at } 313 \mathrm{~K}\left(^{\circ}\right)\end{array}$ & $\operatorname{SEM}\left({ }^{\circ}\right)$ & SD $\left(^{\circ}\right)$ & $\begin{array}{c}\text { Angle of bending } \\
\text { at } 328 \mathrm{~K}\left(^{\circ}\right)\end{array}$ & SEM $\left({ }^{\circ}\right)$ & SD $\left(^{\circ}\right)$ \\
\hline $\mathrm{L}_{10 \mathrm{~nm}}$ & 4.386 & 0.04933 & 2.20613 & 26.081 & 0.05646 & 2.52491 \\
\hline $\mathrm{L}_{30 \mathrm{~nm}}$ & 4.095 & 0.05956 & 2.34862 & 24.777 & 0.05616 & 2.51142 \\
\hline $\mathrm{L}_{50 \mathrm{~nm}}$ & 3.033 & 0.03746 & 1.67528 & 22.152 & 0.06766 & 2.62741 \\
\hline $\mathrm{L}_{70 \mathrm{~nm}}$ & 3.506 & 0.04647 & 1.82597 & 20.742 & 0.06157 & 2.75349 \\
\hline L90nm & 3.315 & 0.04069 & 1.66616 & 18.904 & 0.0682 & 2.5307 \\
\hline $\mathrm{L}_{110 \mathrm{~nm}}$ & 3.849 & 0.046 & 1.81929 & 23.865 & 0.0474 & 2.11963 \\
\hline $\mathrm{L}_{110 \mathrm{~nm}(\Delta 32)}$ & 13.066 & 0.1544 & 6.90483 & 42.616 & 0.0788 & 3.52405 \\
\hline
\end{tabular}

\section{Measurement of forces}

The forces transmitted through the hairpins and linkers can be measured through the forces exerted by the traps modelling their attachment points to the sphere. These traps are harmonic, and so forces they exert have the form $\mathbf{F}=-k(\mathbf{r}-\mathbf{r} 0)$ where $\mathbf{F}$ is the instantaneous force, $k$ is the spring constant or stiffness, $\mathbf{r}$ is the position of the nucleotide at that instant and $\mathbf{r o}$ is the equilibrium position of the trap. If $\mathbf{F}$ is the total force acting on a nucleotide at any instance, the force through the hairpin is measured by defining a component of this force $\mathbf{F}_{\|}$which acts along the direction given by the vector $\mathbf{V}_{\mathbf{3}}=\mathbf{B}_{\mathbf{i}}-\mathbf{D}_{\mathbf{i}}$ where $\mathbf{D}_{\mathbf{i}}$ is the position of the top-most nucleotide in that strand (the nucleotide on which the trap force is being applied). So, we have:

$$
\mathbf{F}_{\|}=\mathbf{F} \cdot \frac{\mathbf{V}_{\mathbf{3}}}{\left\|\mathbf{V}_{\mathbf{3}}\right\|}
$$

We can say that if $\mathbf{F}_{\|}>0$, the linker/hairpin is under tension and if $\mathbf{F}_{\|}<0$, the linker/hairpin is under compression. $\mathbf{F}$ and $\mathbf{F} \|$ were measured at low and high temperatures for each hairpin and they were plotted along with their moving averages (calculated using 11 data points in the neighborhood of a particular data point). The force acting along the hairpins averaged over all configurations and all hairpins in the system is given in Table $\mathbf{S 2}$.

Table S2: Average force and standard error along hairpins $(n=6)$

\begin{tabular}{|c|c|c|c|c|c|c|}
\hline Configuration & $\begin{array}{c}\text { Force along } \\
\text { hairpins at 313 K } \\
(\mathbf{p N})\end{array}$ & $\begin{array}{c}\text { SEM } \\
(\mathbf{p N})\end{array}$ & SD (pN) & $\begin{array}{c}\text { Force along } \\
\text { hairpins at 328 K } \\
(\mathbf{p N})\end{array}$ & $\begin{array}{c}\text { SEM } \\
(\mathbf{p N})\end{array}$ & SD (pN) \\
\hline $\mathrm{L}_{10 \mathrm{~nm}}$ & -0.176 & 0.723 & 1.772 & -0.628 & 0.246 & 0.603 \\
\hline $\mathrm{L}_{30 \mathrm{~nm}}$ & -0.063 & 0.829 & 2.031 & -0.402 & 0.415 & 1.016 \\
\hline $\mathrm{L}_{50 \mathrm{~nm}}$ & -0.229 & 0.644 & 1.577 & -0.075 & 0.588 & 1.441 \\
\hline $\mathrm{L}_{70 \mathrm{~nm}}$ & -0.126 & 0.686 & 1.681 & 0.716 & 0.809 & 1.982 \\
\hline $\mathrm{L}_{90 \mathrm{~nm}}$ & -0.101 & 0.709 & 1.737 & 2.609 & 2.035 & 4.985 \\
\hline $\mathrm{L}_{110 \mathrm{~nm}}(\Delta 32)$ & 0.310 & 0.965 & 2.364 & 4.457 & 1.847 & 4.524 \\
\hline
\end{tabular}

We note that there are fast fluctuations in the instantaneous force due to the trapped nucleotides oscillating around their average position in the trap center; thus, the force distribution is quite wide. At low temperature, we do not observe any of the hairpins breaking. Under high temperature conditions, some of the hairpins break which can be attributed to both thermal and mechanical effects. We clearly observe a trend in hairpin unfolding based upon the position of the hairpin on the origami. The hairpins close to the center of the origami $\left(\mathbf{L}_{\mathbf{1 0}} \mathbf{n m}\right)$ feel the least tension and the ones towards the extremes $\left(\mathbf{L}_{110 n m}\right)$ feel the most tension. From Table S1 it is clear that none of the systems have hairpins under strong tension at $313 \mathrm{~K}$. At $328 \mathrm{~K}$, only hairpins of L110nm experience tension comparable to their $\mathbf{F}_{\mathbf{1} / \mathbf{2}}(\sim 4.8 \mathrm{pN})$. When "mutual traps" are placed onto the 
hairpin nucleotides, they are unable to break and relieve any of the tension. This leads to a small increase in the average force for the systems with hairpins far apart (mainly L90nm and L110nm).

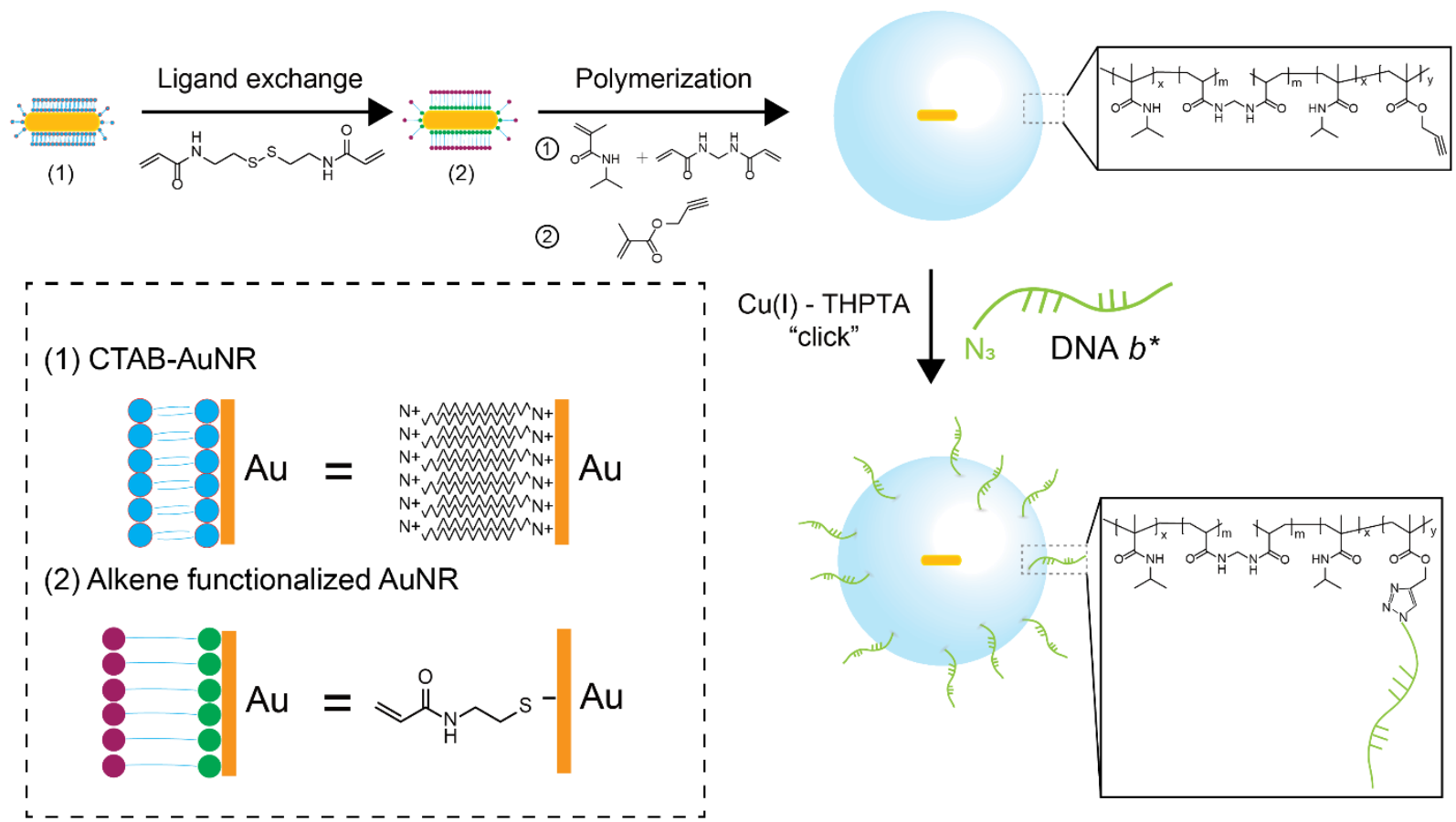

Figure S1. Synthesis of pNIPMAm particle. First, the CTAB-gold nanorod was decorated with an alkene function group (N, N'-bis(acryloyl)cystamine) via ligand exchange. The polymer shell was then built by polymerization of $0.1 \mathrm{~g} \mathrm{~N}$-isopropylmethacrylamide and $0.01 \mathrm{~g}$ of the crosslinking agent N,N'-methylenebisacrylamide at $70^{\circ} \mathrm{C}$ for $2 \mathrm{hr}$. The alkyne functional group was introduced via dropwise injection of $0.01 \mathrm{ml}$ of propargyl methacrylate monomer. DNA anchor was then incorporate on the particle surface via copper-free click chemistry. 

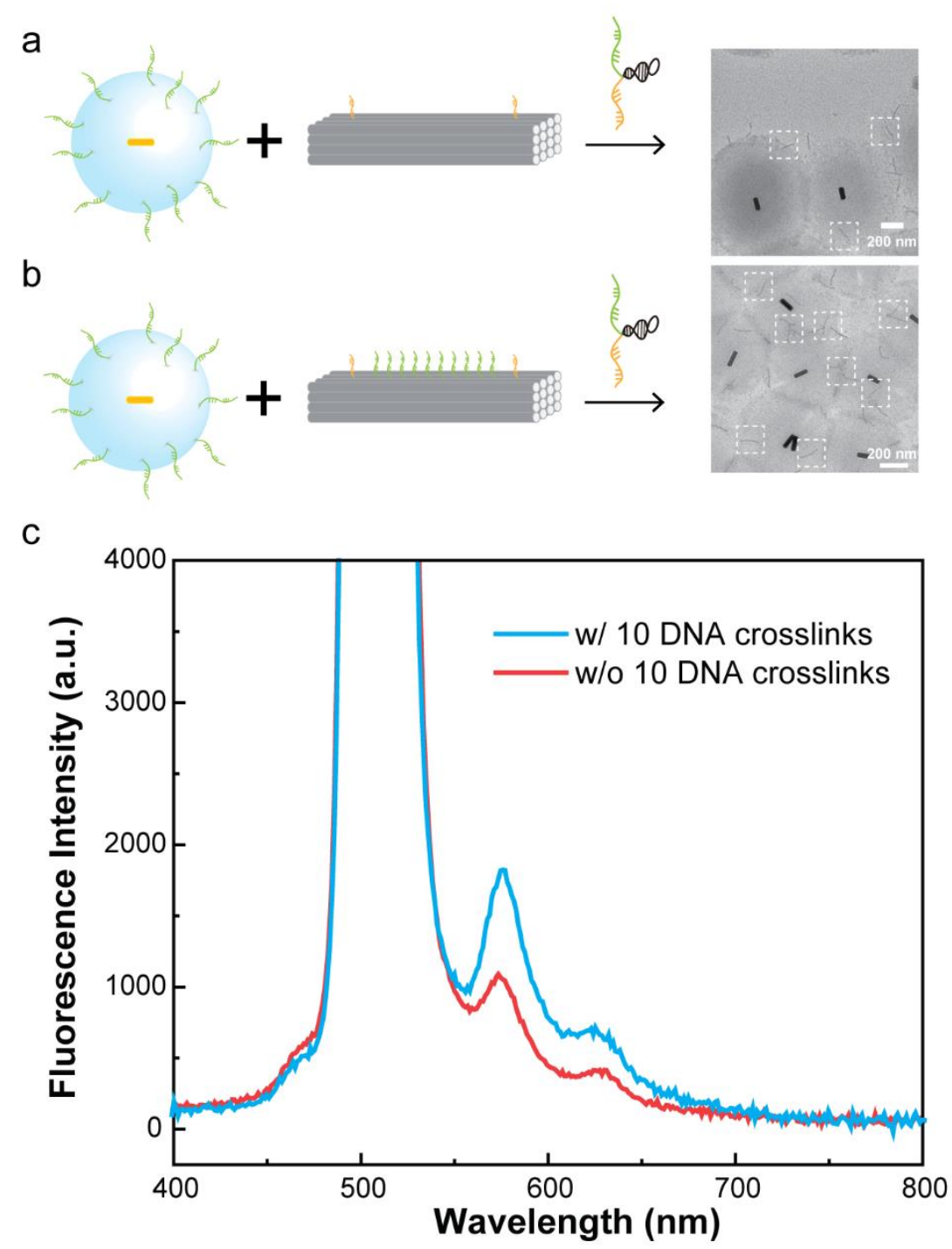

Figure S2. Validating the specificity of DNA-guided OPFC assembly. Schematic and TEM images (a-b) along with fluorescence spectra demonstrating the importance of the 10 DNA crosslinks in boosting the yield of OPFC assembly. The data is representative from two replicates. To reduce the cost of DNA hairpin and origami that were used in replicates, only two hairpins were incorporated on the origami and the particle and DNA origami were mixed in a lower concentration than other OPFC experiments in this work. Fluorescence spectra of nearly identical samples prepared by mixing $8 \mu \mathrm{l}$ of $0.6 \mathrm{nM}$ polymer particles with $50 \mu \mathrm{l}$ of $9 \mathrm{nM}$ origami beam and allowed to hybridize overnight at room temperature. The sample was then washed 5 times (5000 rpm, 6 mins, in $5 \mathrm{mM}$ Tris- $\mathrm{HCl}, 10 \mathrm{mM} \mathrm{MgCl} 2$ and $1 \mathrm{mM}$ EDTA at $\mathrm{pH}=8$ ) by centrifugation and redispersed for experiments. The fluorescence spectra in (c) were collected using a $520 \mathrm{~nm}$ excitation wavelength that was selected to excite the Cy3B dye while also providing some spectral separation from the strong scattering generated by the responsive particles. The Cy3B emission peak was at $575 \mathrm{~nm}$ and its intensity indicates the yield of OPFCs assembled with origami beams. 

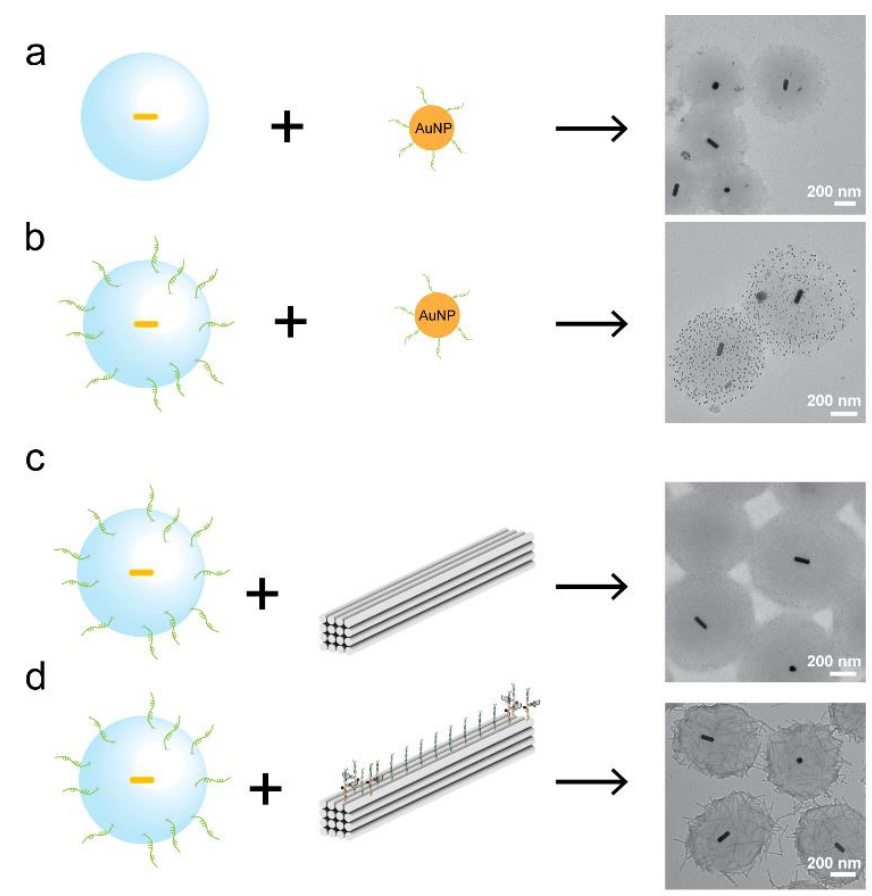

e

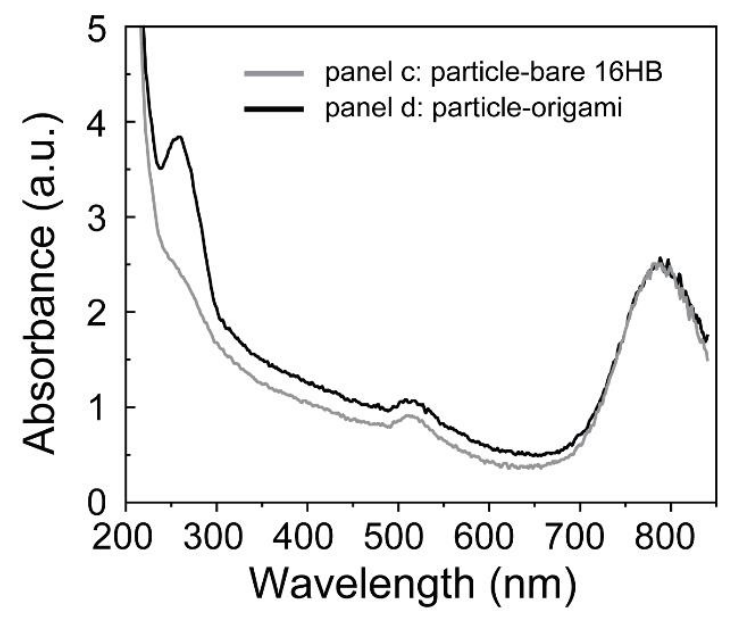

Figure S3. Characterization and validation of DNA-mediated OPFC assembly. (a-b): Schematic and TEM images of pNIPMAm particles with/without DNA handles (b-b*) incubated with DNA coated AuNPs. (c-d): Schematic and TEM images of pNIPMAm particles incubated with 16HB DNA origami loaded with/without target molecules. (e): Absorbance spectra of panel $\mathrm{c}$ and d confirms DNA-mediated assembly of OPFC. Absorbance spectra were collected using NanoDrop 2000 (Thermo Scientific) from $200 \mathrm{~nm}$ to $850 \mathrm{~nm}$. Samples were prepared by mixing $15 \mu \mathrm{l}$ of $0.3 \mathrm{nM}$ of polymer particles with $50 \mu \mathrm{l}$ of $30 \mathrm{nM}$ origami beam or $50 \mu \mathrm{l}$ of $\sim 1 \mathrm{nM} 13 \mathrm{~nm}$ lab-synthesized gold nanoparticles and allowed to hybridize overnight at room temperature. The sample was then washed 5 times $(5000 \mathrm{rpm}, 6$ mins, in $5 \mathrm{mM}$ Tris- $\mathrm{HCl}, 10 \mathrm{mM} \mathrm{MgCl} 2$ and $1 \mathrm{mM}$ EDTA at $\mathrm{pH}=8)$ by centrifugation and redispersed in $40 \mu \mathrm{l}$ buffer B $(5 \mathrm{mM}$ Tris- $\mathrm{HCl}, 10 \mathrm{mM}$ $\mathrm{MgCl}_{2}$ and $1 \mathrm{mM}$ EDTA at $\mathrm{pH}=8$ ) and measured shortly after sample preparation. 
a
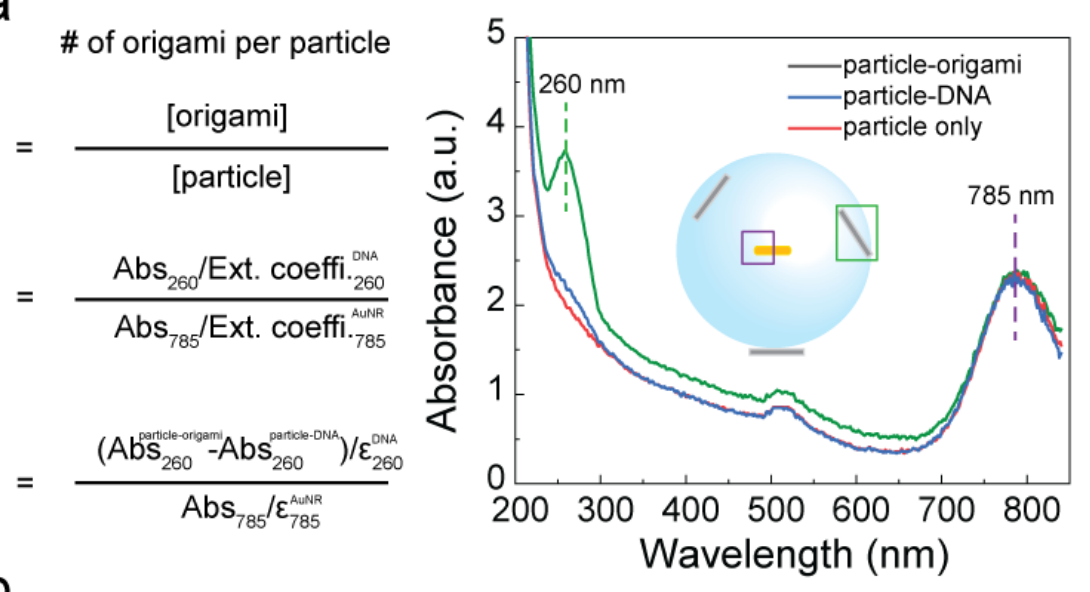

b

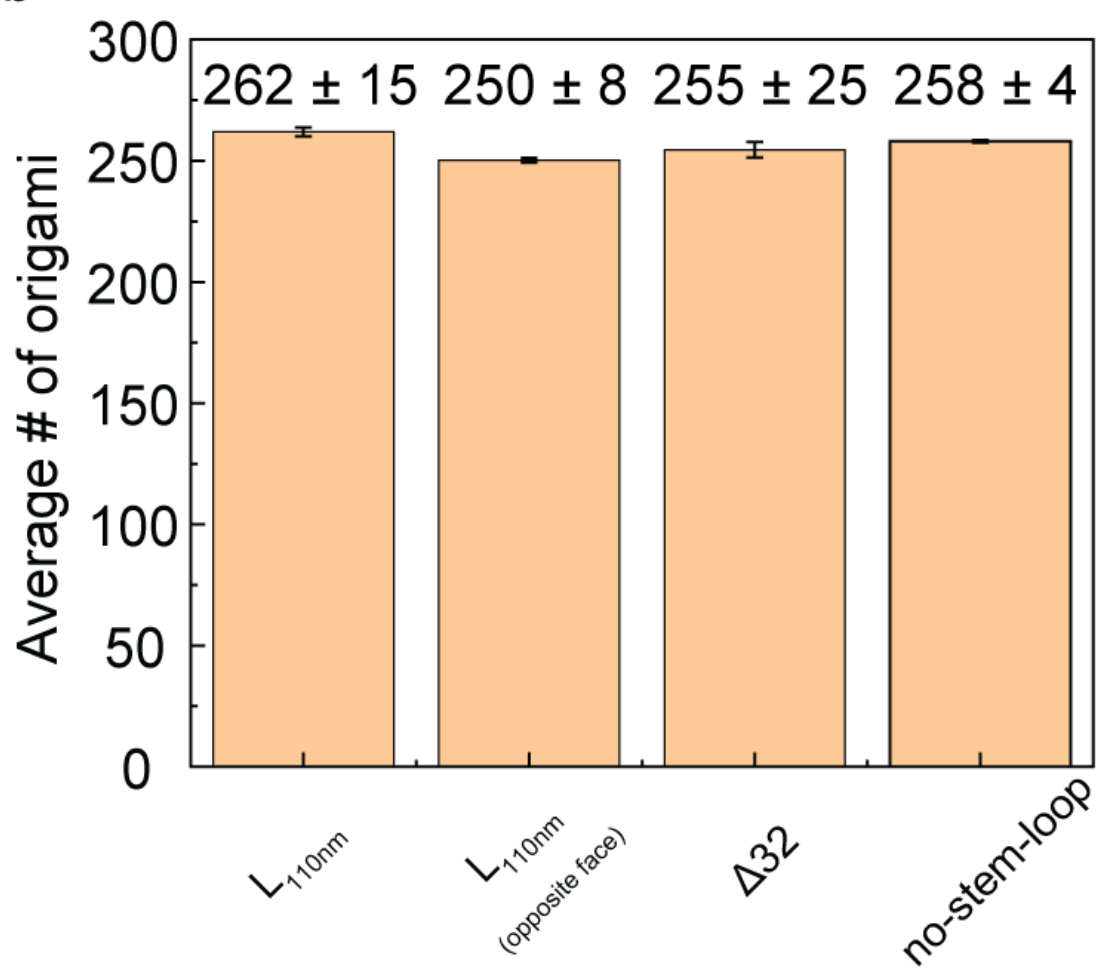

Figure S4. Quantification of origami beam number on polymer particles. (a) Absorbance spectroscopy of OPFC and controls. Stoichiometry between origami and particle is quantified using the extinction coefficient of the AuNR core within each OPFC $\left(\varepsilon 785 \mathrm{~nm}=3.7 \times 10^{10} \mathrm{M}^{-1} \mathrm{~cm}^{-1}\right)$ and the origami $\left(\varepsilon_{260 \mathrm{~nm}}=1 \times 10^{8} \mathrm{M}^{-1} \mathrm{~cm}^{-1}\right)$. Absorbance at $260 \mathrm{~nm}\left(\mathrm{Abs}_{260}\right)$ was corrected by subtracting the absorbance at $260 \mathrm{~nm}$ of particle-DNA (blue curve, Abs $260^{\text {particle-DNA) from particle- }}$ origami sample (green curve, Abs260 ${ }^{\text {particle-origami }}$ ). (b) Plots of origami beam copy number on each OPFC in different OPFC designs. Error bars represent the standard deviation from three independent measurements. Samples were prepared as described in Figure S3. $2 \mu 1$ of each sample was added to NanoDrop 2000 spectrophotometer for absorbance measurement. 

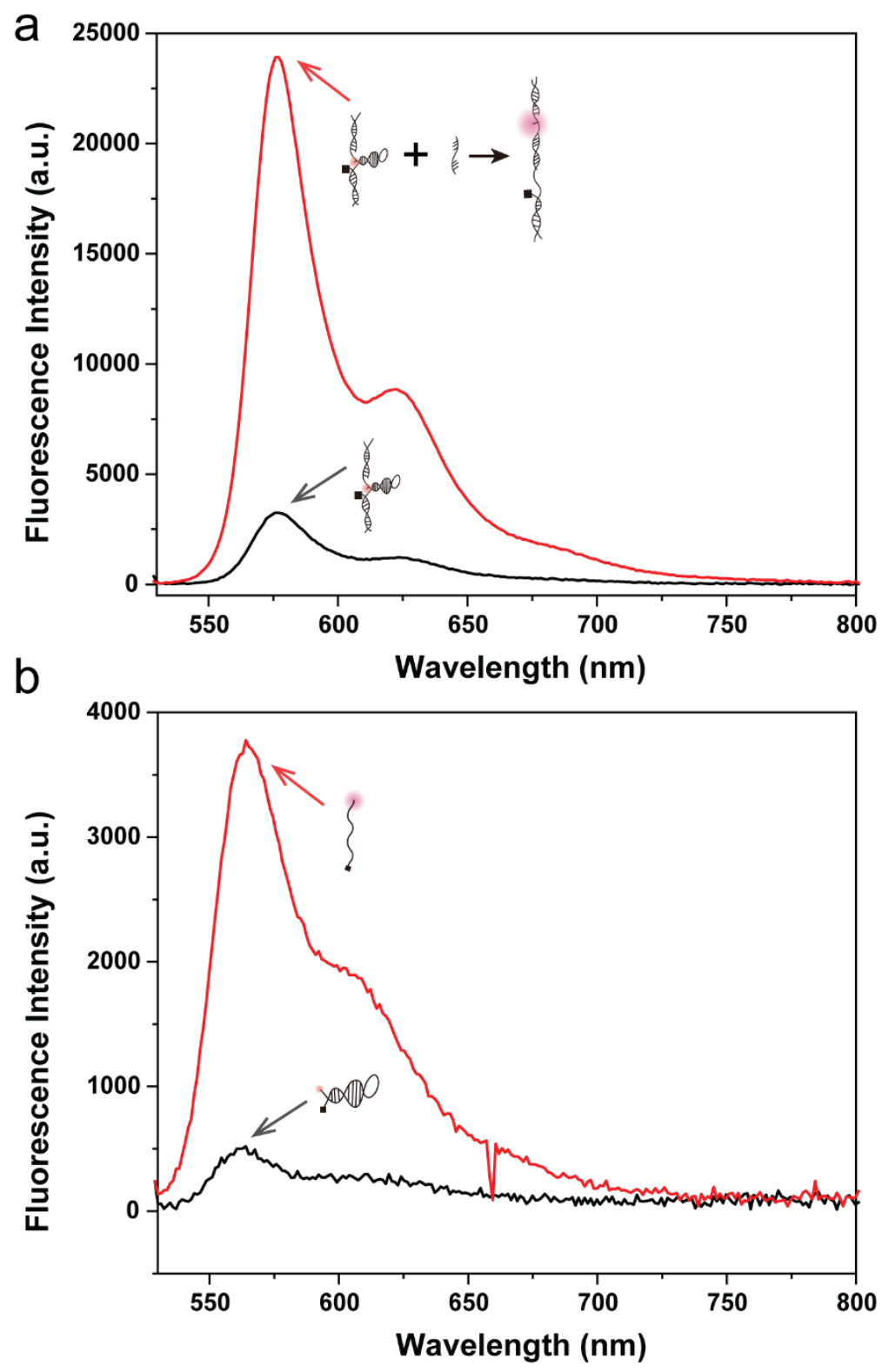

Figure S5. Fluorescence measurements of DNA hairpin in folded and extended states. (a) Spectra showing the fluorescence intensity of DNA hairpin before (black) and after (red) hybridizing to a 17 mer short oligonucleotide (sequence shown in Table S1). The fluorophore (Cy3B) and quencher (QSY9) pair positioned on complementary strands of the hairpin. To unfold the DNA hairpin, $150 \mathrm{nM}$ of DNA hairpin was hybridized with $1.5 \mu \mathrm{M} 17$ mer complementary DNA for $3 \mathrm{hr}$ at room temperature. (b) Spectra showing the fluorescence intensity of hairpins lacking the arms and only including the stem region before (black, $20^{\circ} \mathrm{C}$ ) and after $\left(\mathrm{red}, 70^{\circ} \mathrm{C}\right.$ ) thermal melting. The fluorophore (Cy3) and quencher (QSY9) pair positioned at the two ends of the hairpin. Both samples were prepared and measured at $150 \mathrm{nM}$ in Buffer B $(5 \mathrm{mM}$ Tris- $\mathrm{HCl}$, $10 \mathrm{mM} \mathrm{MgCl} 2$ and $1 \mathrm{mM}$ EDTA at $\mathrm{pH}=8$ ) and measured shortly after sample preparation. The significantly different maximum intensity between $\mathbf{a}$ and $\mathbf{b}$ is likely due to the different quantum yield of $\mathrm{Cy} 3 \mathrm{~B}$ and $\mathrm{Cy} 3$. ( $\mathrm{Cy} 3 \mathrm{~B}$ is reported having $\sim 8$ fold greater fluorescence compared to $\mathrm{Cy} 3$ at equimolar concentration ${ }^{25}$ ) 
a

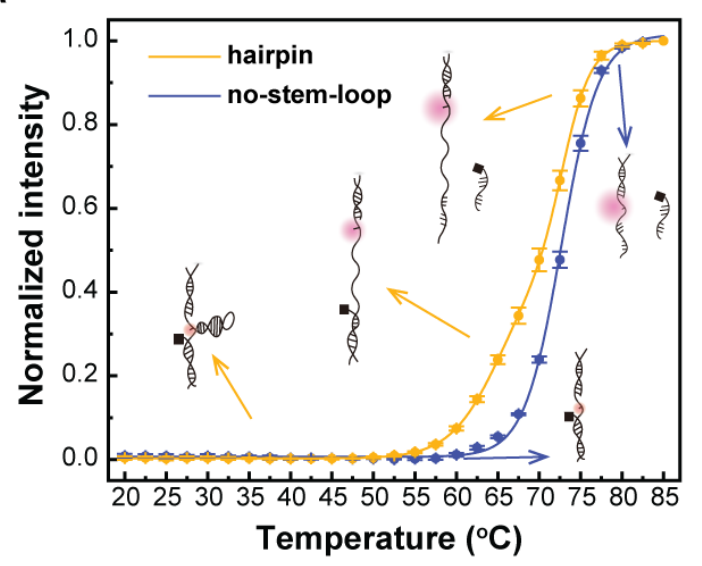

C

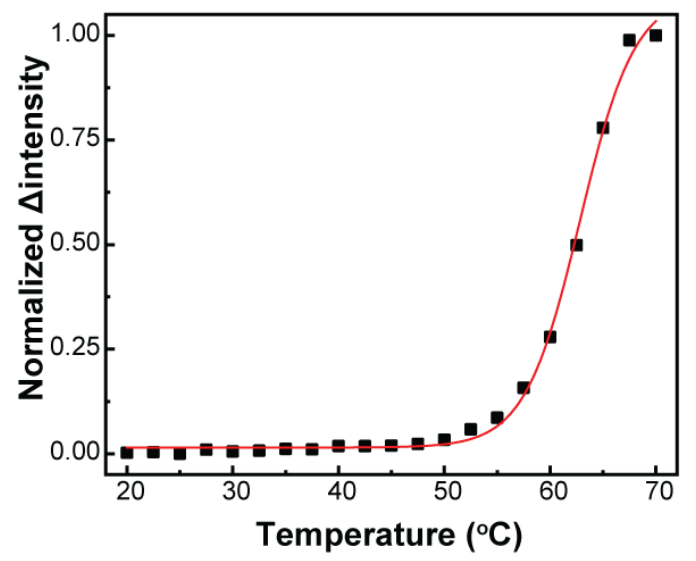

b

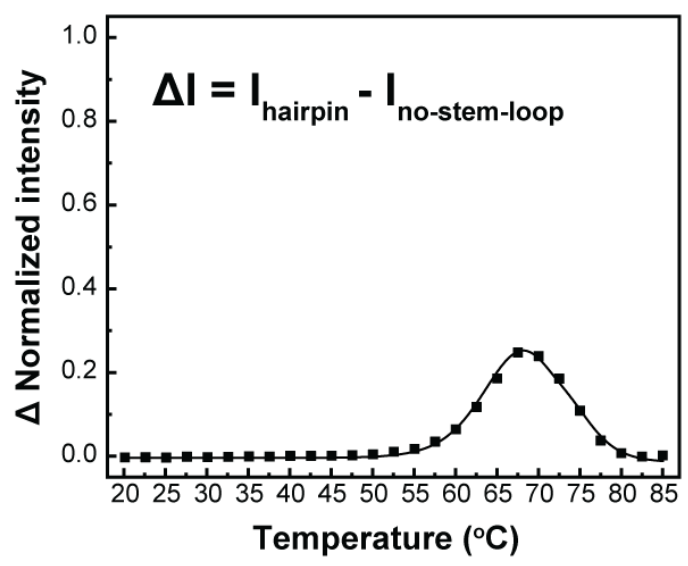

d

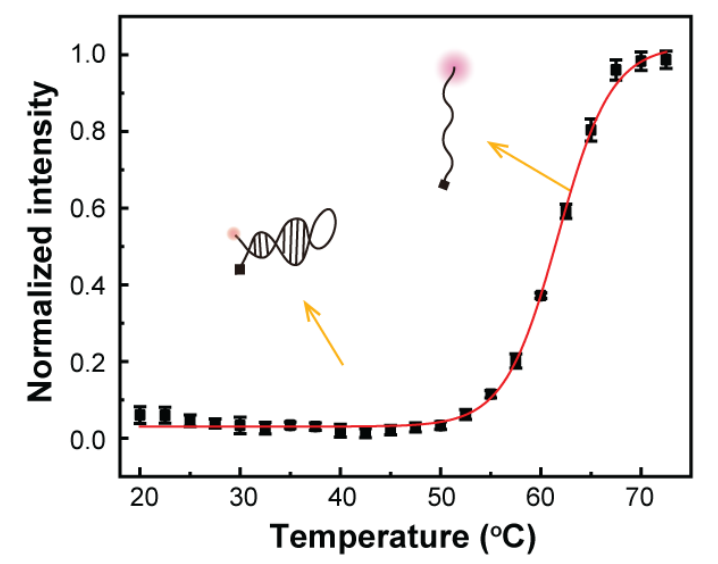

Figure S6. Thermal melting profile of target DNA hairpins. (a) Temperature-dependent fluorescence intensity of DNA hairpin and control lacking the stem-loop domain. The blue represents a control lacking the stem-loop while the yellow corresponds to the standard $4.7 \mathrm{pN}$ hairpin used throughout this study. The increase in fluorescence is due to both the stem melting as well as arm melting, and these two transitions are overlapping with stem $\mathrm{T}_{\mathrm{m}}=64^{\circ} \mathrm{C}$ and $\operatorname{arm~} \mathrm{T}_{\mathrm{m}}=$ $72^{\circ} \mathrm{C}$. Error bars correspond to the standard deviation of triplicate measurements. (b) Fluorescence intensity difference between hairpin and no-stem loop control as measured from the thermal melting experiments. (c) A normalized plot using the data from panel $\mathbf{B}$ in the range from $20^{\circ} \mathrm{C}$ to $70^{\circ} \mathrm{C}$. (d) Temperature-dependent fluorescence intensity of DNA hairpins identical to the one studied through this work but lacking the arms. The $\mathrm{T}_{\mathrm{m}}$ here was $=62^{\circ} \mathrm{C}$ confirming minimal contribution from the arms. For all these measurement, [DNA] $=150 \mathrm{nM}$ and the hairpin was prepared from a 1:1:1 mixture of the three oligonucleotides comprising the hairpin probe. Measurements were performed in Buffer B (5 mM Tris- $\mathrm{HCl}, 10 \mathrm{mM} \mathrm{MgCl} 2$ and $1 \mathrm{mM}$ EDTA, pH $=8)$. 

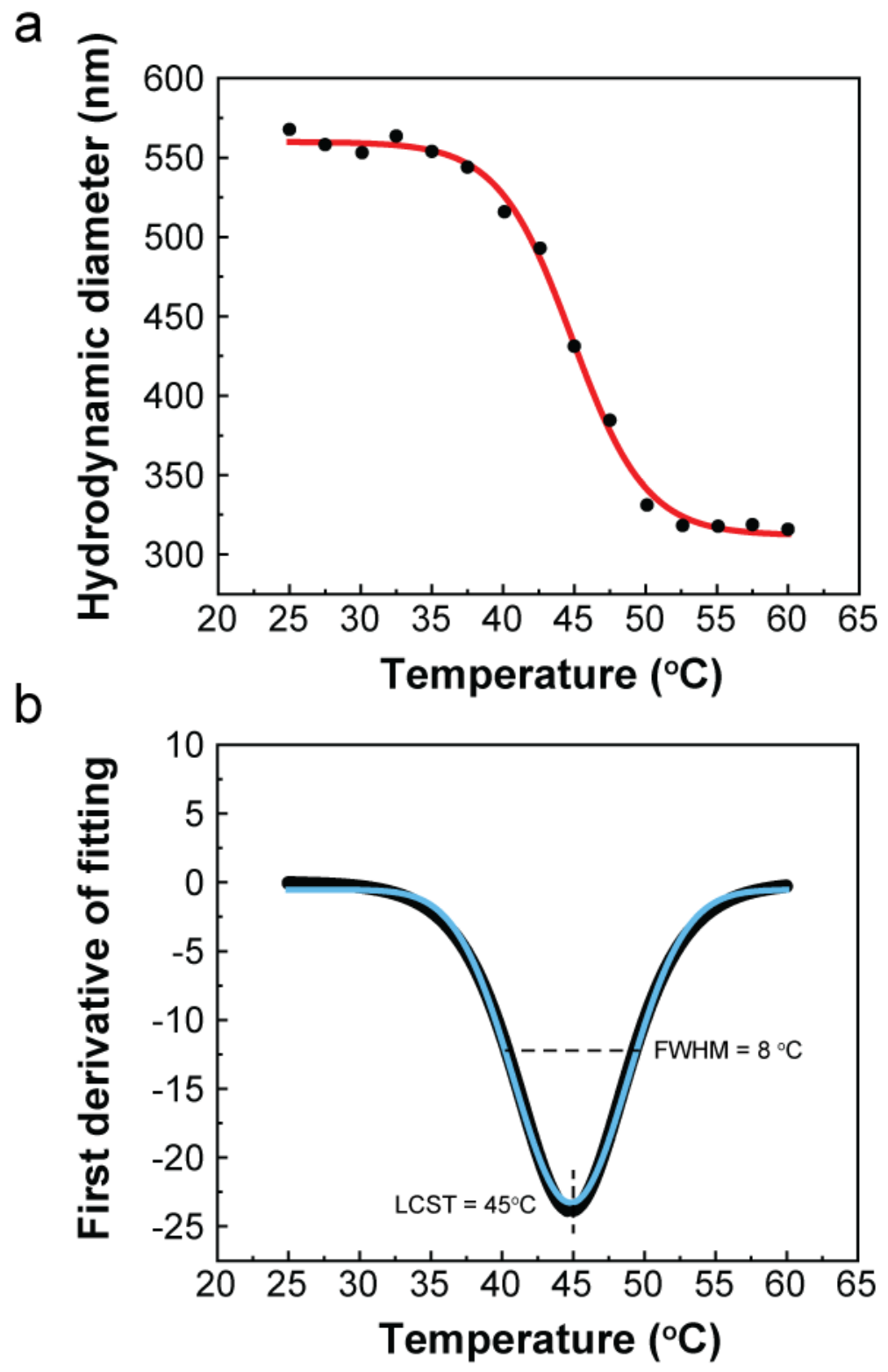

Figure S7. Characterization of transition temperature of particle actuators. (a) Hydrodynamic diameters of particles as a function of temperature. The data was fit with a Boltzmann sigmoid function. (b) Calculated first derivative of the data shown in a, confirming transition temperature $\mathrm{LCST}=45^{\circ} \mathrm{C}$ and $\mathrm{FWHM}=8^{\circ} \mathrm{C}$. 

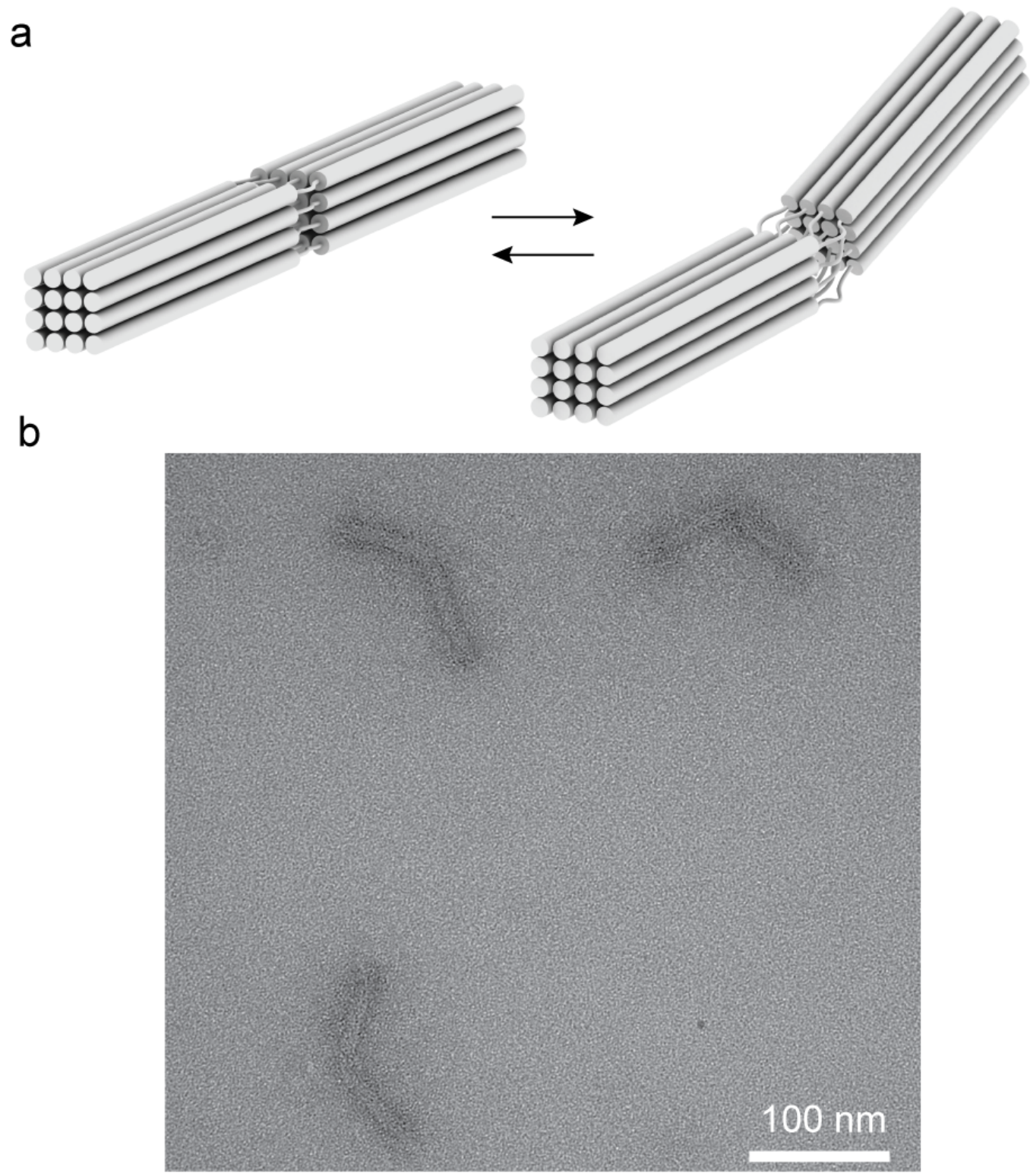

Figure S8. Design of flexible origami beams $(\mathbf{\Delta 3 2})$. (a) Schematic showing the $\Delta 32$ origami beam. The deletion of 16 staple strands in the center of the origamis gives the structure significantly more flexibility. (b) Representative TEM image showing bending of $\Delta 32$ structures. 
a

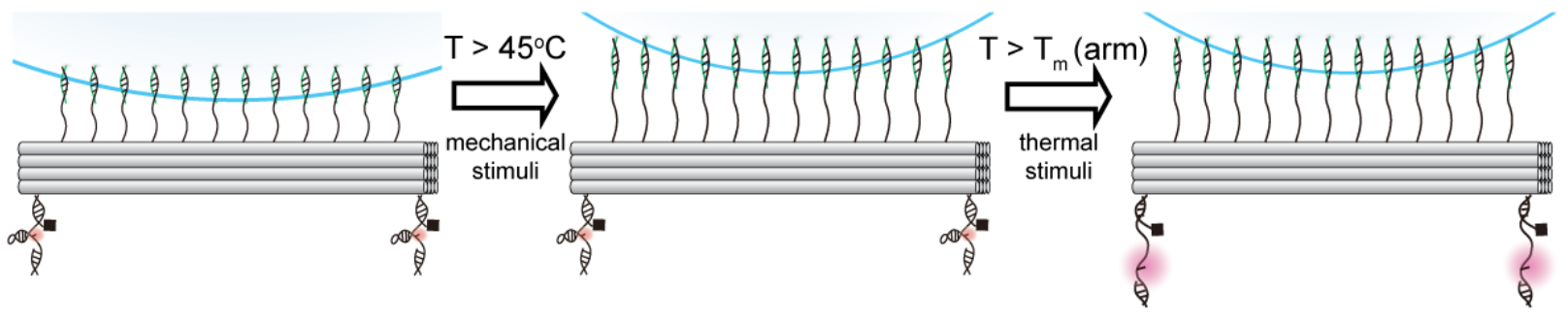

b
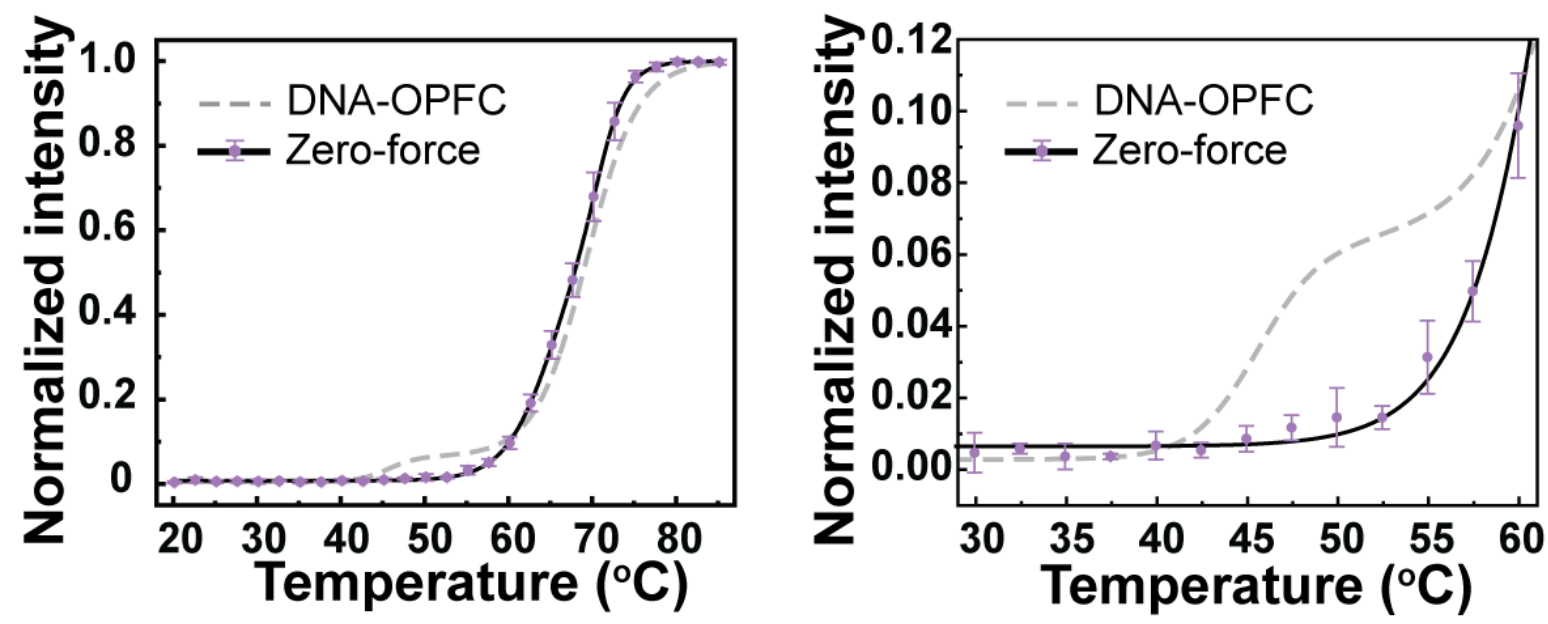

Figure S9. Zero-force control OPFC device with target hairpins located at the opposite face away from the particle actuator. (a) Schematics of zero-force control. In this experiment, we created chemically identical OPFC loaded with the same target, but with a geometric mutation. The hairpin was placed on the opposite face of the beam away from the 10 crosslinks and the particle. In this case, the collapse of the particle is not expected to generate any mechanical tension on the hairpin. (b) Bulk temperature-dependent fluorescence measurements of zero-force control showed no notable mechanical unfolding signal in the $45-55^{\circ} \mathrm{C}$ window. Samples were prepared by mixing $15 \mu 1$ of $0.3 \mathrm{nM}$ of polymer particles with $50 \mu 1$ of $30 \mathrm{nM}$ origami beam. The mixture was then washed by centrifuge and redispersed in $40 \mu 1$ Buffer B as described before in the methods section. Error bars represent the standard deviation from triplicate preparations of the zero-force control OPFC. The black line is the same fit as the DNA-OPFC data shown in Fig. 1d (blue curve). 

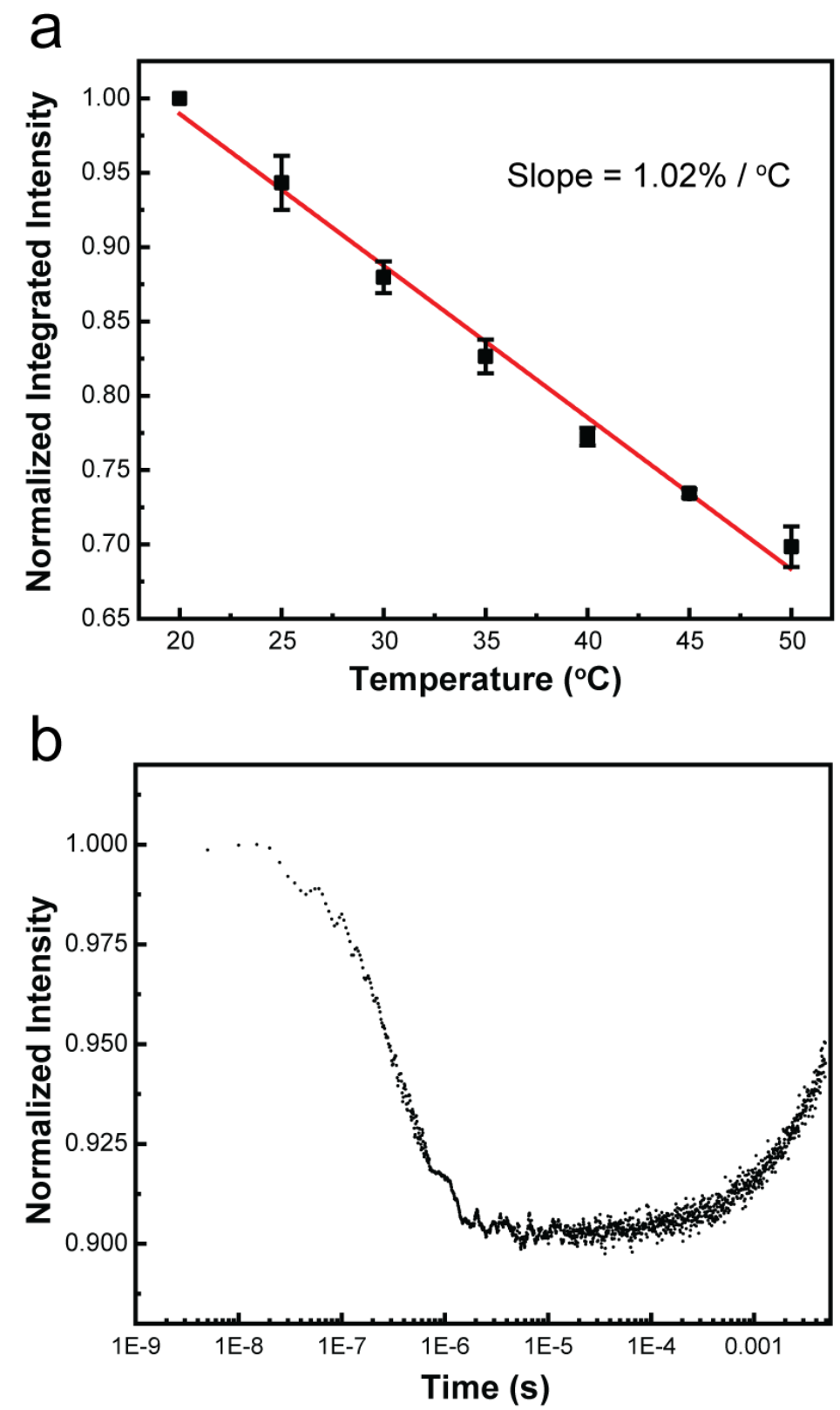

Figure S10. Temperature calibration curve of T-jump experiment with Rhodamine B standard. (a) Calibration curve measuring the temperature-dependent emission of Rhodamine B at a concentration $=150 \mathrm{nM}$. The temperature was ramped from 20 to $50{ }^{\circ} \mathrm{C}$ and controlled using a water bath and allowed to come to equilibrium for $5 \mathrm{~min}$ at each temperature. Error bars represent the standard deviation from triplicate measurements. The red line represents a linear regression to the data (Intensity $=-0.0102 *$ Temperature $+1.1038, \mathrm{R}^{2}=0.9907$ ). (b) Time-resolved fluorescence signal of Rhodamine B following a 10 nsec pump. The plot shows a 10\% reduction of fluorescence intensity, which indicates a $10^{\circ} \mathrm{C}$ temperature jump, and confirms the $3 \mu$ s instrument response time. 


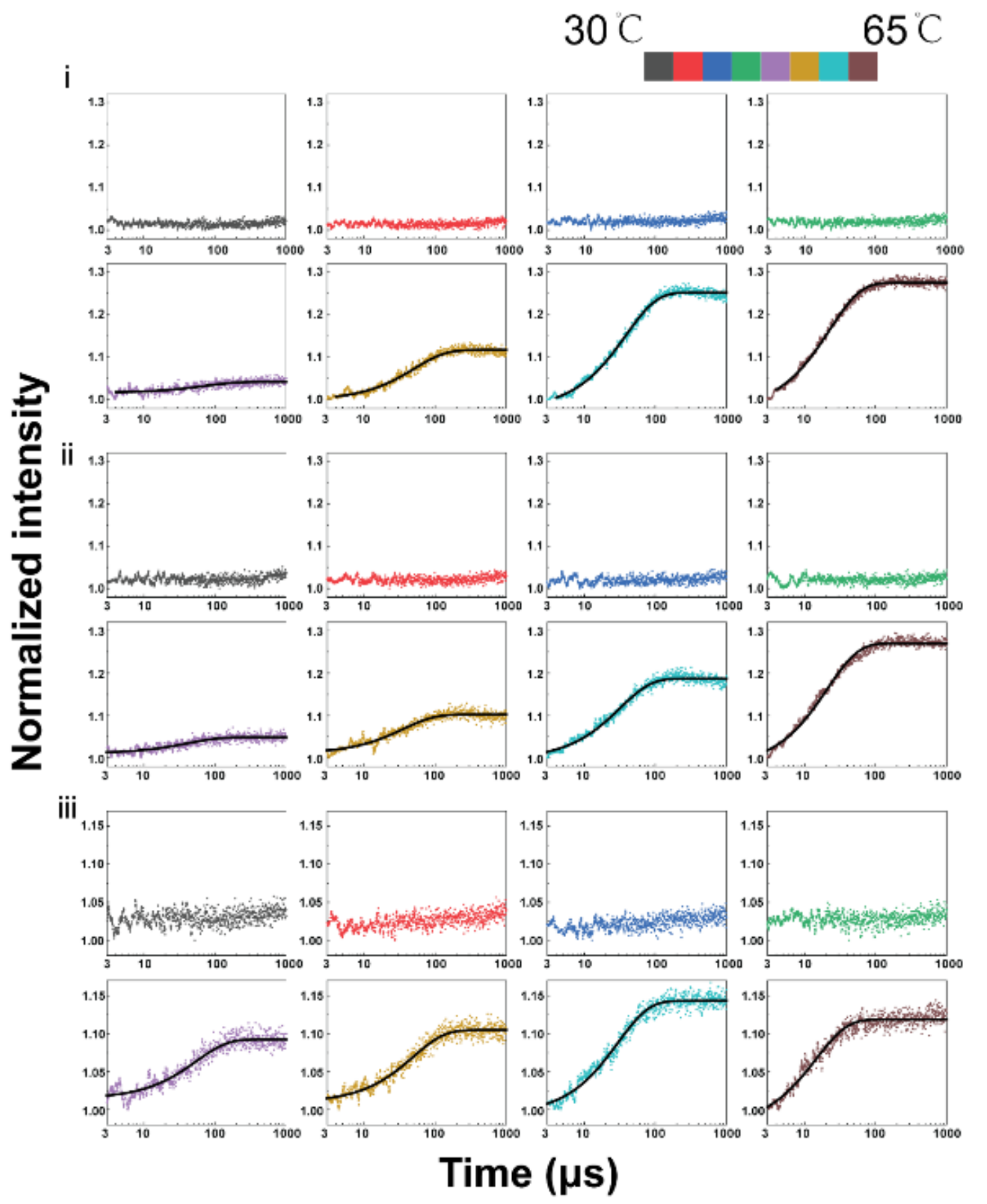

Figure S11. Spectra of T-jump experiments. Three replicates (i)-(iii) of temperature jump $(\Delta T$ $\left.=10^{\circ} \mathrm{C}\right)$ showing transients of DNA hairpin $(100 \mathrm{nM})$ thermal melting in buffer $\mathrm{B}$. The data is fitted to a single exponential (black line). The colors indicate the initial temperature of the sample and ranged from $30^{\circ} \mathrm{C}$ to $65^{\circ} \mathrm{C}$. Importantly, there was no thermal melting signal when the initial temperature was $45^{\circ} \mathrm{C}$ or lower. This is consistent with the bulk fluorescence data showing that the $\mathrm{T}_{\mathrm{m}}$ of the hairpins is $64^{\circ} \mathrm{C}$. 

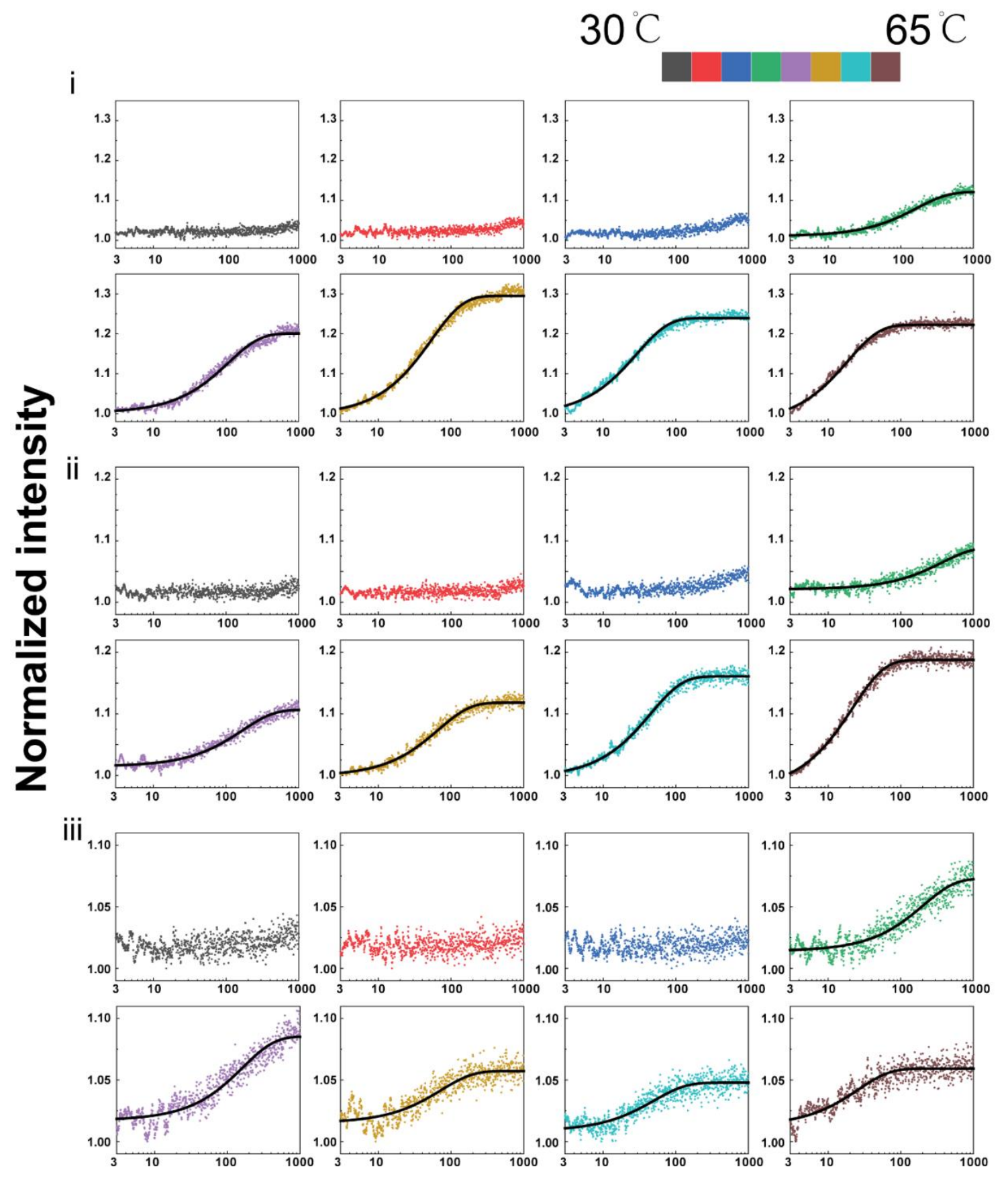

\section{Time ( $\mu s)$}

Figure S12. Spectra of F-jump experiments. Three replicates (i)-(iii) of force jump measurements $\left(\Delta \mathrm{T}=10^{\circ} \mathrm{C}\right)$ showing transients of mechanical unfolding of DNA hairpins $(\sim 100$ $\mathrm{nM}$ ) loaded onto the OPFC. The data is fitted to a single exponential (black line). The colors indicate the initial temperature of the sample and ranged from $30^{\circ} \mathrm{C}$ to $65^{\circ} \mathrm{C}$. Importantly, there is now a mechanical melting signal when the initial temperature was $45^{\circ} \mathrm{C}$ which is consistent with the LCST of pNIPMAm. 


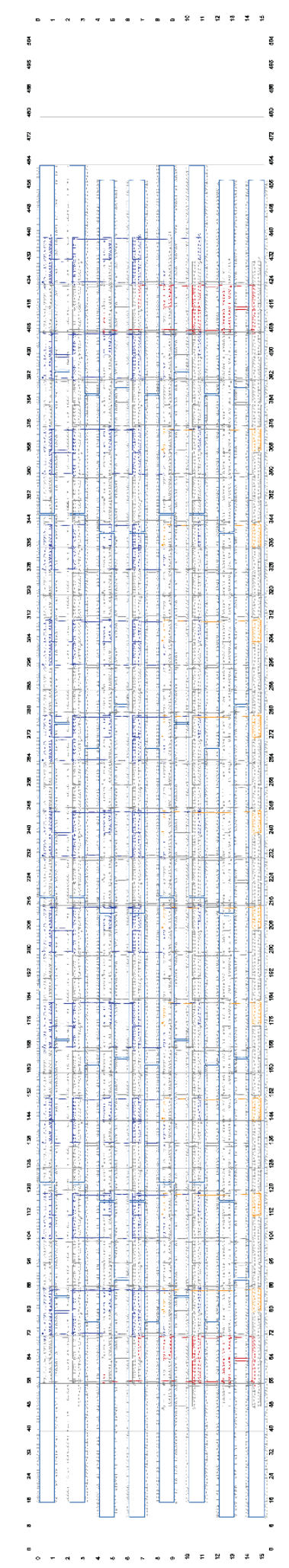

Figure S13. Schematic of the 16HB origami generated by caDNAno. Blue: p7560 scaffold strand, Red: hairpin binding staples. Orange: particle binding staples. Additional information showing full staple sequences can be found in Table S1-3. 
Table S1.

Oligonucleotide ID and sequences used in this work.

\begin{tabular}{|c|c|}
\hline Strand ID & DNA Sequences (5' to 3') \\
\hline $\begin{array}{l}\text { particle-linker } \\
\text { (sequence } b^{*} \text { ) }\end{array}$ & GCA GTG TGT GAG TGG TTT CAG TTT /3AzideN/ \\
\hline hairpin & $\begin{array}{l}\text { CTG AAA CCA CTC ACA CAC TGC /iUniAmM/ GTA TAA ATG } \\
\text { TTT TTT TCA TTT ATA C AGC GCC ACG TAG CCC AGC }\end{array}$ \\
\hline no-stem-loop & $\begin{array}{l}\text { CTG AAA CCA CTC ACA CAC TGC/iUniAmM/AGC GCC ACG } \\
\text { TAG CCC AGC }\end{array}$ \\
\hline 17-mer & GAA AAA AAC ATT TAT AC \\
\hline $\begin{array}{c}\text { anchor } 1 \\
\text { (sequence } \mathrm{a}^{*} \text { ) }\end{array}$ & $\begin{array}{l}\text { TTTCAGGGCCACCCTCCGATTGGCGAATCAAGGTCACCAA- } \\
\text { TT-GCTGGGCTACGTGGCGCT }\end{array}$ \\
\hline $\begin{array}{c}\text { anchor } 2 \\
\text { (sequence } \mathrm{a}^{*} \text { ) }\end{array}$ & $\begin{array}{l}\text { CGGAACCTGTTTTAACGCCAGAATCCGGAAACTTTGCCTT- } \\
\text { TT-GCTGGGCTACGTGGCGCT }\end{array}$ \\
\hline $\begin{array}{c}\text { anchor } 3 \\
\text { (sequence } \mathrm{a}^{*} \text { ) }\end{array}$ & $\begin{array}{l}\text { AGGTCAGAAGAACCGCTTCATAATCAAAATCA-TT- } \\
\text { GCTGGGCTACGTGGCGCT }\end{array}$ \\
\hline $\begin{array}{c}\text { anchor } 4 \\
\left(\text { sequence } a^{*}\right)\end{array}$ & $\begin{array}{l}\text { GCGTTATAGCACCCAGCCGGTATTCGTCAAAAGACGGGAG- } \\
\text { TT-GCTGGGCTACGTGGCGCT }\end{array}$ \\
\hline $\begin{array}{c}\text { anchor } 5 \\
\text { (sequence } \mathrm{a}^{*} \text { ) }\end{array}$ & $\begin{array}{l}\text { AGCATGTAAATAATCGTACCGCGCGCGCATTAATGAAAAT- } \\
\text { TT-GCTGGGCTACGTGGCGCT }\end{array}$ \\
\hline $\begin{array}{c}\text { anchor } 6 \\
\left.\text { (sequence } \mathrm{a}^{*}\right)\end{array}$ & $\begin{array}{l}\text { AGGCTTATCTACAATTTCTTACCAACGCTAAC-TT- } \\
\text { GCTGGGCTACGTGGCGCT }\end{array}$ \\
\hline $\begin{array}{c}\text { crosslink } 1 \\
\text { (sequence b) }\end{array}$ & $\begin{array}{l}\text { CTGAAACCACTCACACACTGC-TT- } \\
\text { ACATAAAAAATAGCTAAACAGTAC }\end{array}$ \\
\hline $\begin{array}{l}\text { crosslink } 2 \\
\text { (sequence b) }\end{array}$ & $\begin{array}{l}\text { CTGAAACCACTCACACACTGC-TT- } \\
\text { AACGTTATAAGTAAGCTTTTCAGG }\end{array}$ \\
\hline $\begin{array}{c}\text { crosslink } 3 \\
\text { (sequence b) }\end{array}$ & $\begin{array}{l}\text { CTGAAACCACTCACACACTGC-TT- } \\
\text { AATTCGACAGGAAACCCAGAAGAT }\end{array}$ \\
\hline $\begin{array}{c}\text { crosslink } 4 \\
\text { (sequence b) }\end{array}$ & $\begin{array}{l}\text { CTGAAACCACTCACACACTGC-TT- } \\
\text { TATGATACACCCAAAAGTGCCTAA }\end{array}$ \\
\hline $\begin{array}{c}\text { crosslink } 5 \\
\text { (sequence b) }\end{array}$ & $\begin{array}{l}\text { CTGAAACCACTCACACACTGC-TT- } \\
\text { CTAATCTAATTACGCAACGAGCCG }\end{array}$ \\
\hline $\begin{array}{l}\text { crosslink } 6 \\
\text { (sequence b) }\end{array}$ & $\begin{array}{l}\text { CTGAAACCACTCACACACTGC-TT- } \\
\text { TATGACAACATACATAATCTGCCA }\end{array}$ \\
\hline $\begin{array}{l}\text { crosslink } 7 \\
\text { (sequence b) }\end{array}$ & $\begin{array}{l}\text { CTGAAACCACTCACACACTGC-TT- } \\
\text { CTTAAGTGGCAAAGACATATCGCG }\end{array}$ \\
\hline $\begin{array}{c}\text { crosslink } 8 \\
\text { (sequence b) }\end{array}$ & $\begin{array}{l}\text { CTGAAACCACTCACACACTGC-TT- } \\
\text { GTCAGGACACAATCAAAAAAGATT }\end{array}$ \\
\hline $\begin{array}{c}\text { crosslink } 9 \\
\text { (sequence b) }\end{array}$ & $\begin{array}{l}\text { CTGAAACCACTCACACACTGC-TT- } \\
\text { TATGCGATCGCCAAAGTATTATAG }\end{array}$ \\
\hline $\begin{array}{l}\text { crosslink } 10 \\
\text { (sequence b) }\end{array}$ & $\begin{array}{l}\text { CTGAAACCACTCACACACTGC-TT- } \\
\text { TTAATTTCTGAGGGAGTGTGTCGA }\end{array}$ \\
\hline
\end{tabular}


Table S2.

Origami scaffold sequence (p7560)

AGCTTGGCACTGGCCGTCGTTTTACAACGTCGTGACTGGGAAAACCCTGGCGTTACCCAA CTTAATCGCCTTGCAGCACATCCCCCTTTCGCCAGCTGGCGTAATAGCGAAGAGGCCCGC ACCGATCGCCCTTCCCAACAGTTGCGCAGCCTGAATGGCGAATGGCGCTTTGCCTGGTTTC CGGCACCAGAAGCGGTGCCGGAAAGCTGGCTGGAGTGCGATCTTCCTGAGGCCGATACTG TCGTCGTCCCCTCAAACTGGCAGATGCACGGTTACGATGCGCCCATCTACACCAACGTGA CCTATCCCATTACGGTCAATCCGCCGTTTGTTCCCACGGAGAATCCGACGGGTTGTTACTC GCTCACATTTAATGTTGATGAAAGCTGGCTACAGGAAGGCCAGACGCGAATTATTTTTGA TGGCGTTCCTATTGGTTAAAAAATGAGCTGATTTAACAAAAATTTAATGCGAATTTTAACA AAATATTAACGTTTACAATTTAAATATTTGCTTATACAATCTTCCTGTTTTTGGGGCTTTTC TGATTATCAACCGGGGTACATATGATTGACATGCTAGTTTTACGATTACCGTTCATCGATT CTCTTGTTTGCTCCAGACTCTCAGGCAATGACCTGATAGCCTTTGTAGATCTCTCAAAAAT AGCTACCCTCTCCGGCATTAATTTATCAGCTAGAACGGTTGAATATCATATTGATGGTGAT TTGACTGTCTCCGGCCTTTCTCACCCTTTTGAATCTTTACCTACACATTACTCAGGCATTGC ATTTAAAATATATGAGGGTTCTAAAAATTTTTATCCTTGCGTTGAAATAAAGGCTTCTCCC GCAAAAGTATTACAGGGTCATAATGTTTTTGGTACAACCGATTTAGCTTTATGCTCTGAGG CTTTATTGCTTAATTTTGCTAATTCTTTGCCTTGCCTGTATGATTTATTGGATGTTAATGCTA CTACTATTAGTAGAATTGATGCCACCTTTTCAGCTCGCGCCCCAAATGAAAATATAGCTAA ACAGGTTATTGACCATTTGCGAAATGTATCTAATGGTCAAACTAAATCTACTCGTTCGCAG AATTGGGAATCAACTGTTATATGGAATGAAACTTCCAGACACCGTACTTTAGTTGCATATT TAAAACATGTTGAGCTACAGCATTATATTCAGCAATTAAGCTCTAAGCCATCCGCAAAAA TGACCTCTTATCAAAAGGAGCAATTAAAGGTACTCTCTAATCCTGACCTGTTGGAGTTTGC TTCCGGTCTGGTTCGCTTTGAAGCTCGAATTAAAACGCGATATTTGAAGTCTTTCGGGCTT CCTCTTAATCTTTTTGATGCAATCCGCTTTGCTTCTGACTATAATAGTCAGGGTAAAGACCT GATTTTTGATTTATGGTCATTCTCGTTTTCTGAACTGTTTAAAGCATTTGAGGGGGATTCAA TGAATATTTATGACGATTCCGCAGTATTGGACGCTATCCAGTCTAAACATTTTACTATTAC CCCCTCTGGCAAAACTTCTTTTGCAAAAGCCTCTCGCTATTTTGGTTTTTATCGTCGTCTGG TAAACGAGGGTTATGATAGTGTTGCTCTTACTATGCCTCGTAATTCCTTTTGGCGTTATGTA TCTGCATTAGTTGAATGTGGTATTCCTAAATCTCAACTGATGAATCTTTCTACCTGTAATA ATGTTGTTCCGTTAGTTCGTTTTATTAACGTAGATTTTTCTTCCCAACGTCCTGACTGGTAT AATGAGCCAGTTCTTAAAATCGCATAAGGTAATTCACAATGATTAAAGTTGAAATTAAAC CATCTCAAGCCCAATTTACTACTCGTTCTGGTGTTTCTCGTCAGGGCAAGCCTTATTCACTG AATGAGCAGCTTTGTTACGTTGATTTGGGTAATGAATATCCGGTTCTTGTCAAGATTACTC TTGATGAAGGTCAGCCAGCCTATGCGCCTGGTCTGTACACCGTTCATCTGTCCTCTTTCAA AGTTGGTCAGTTCGGTTCCCTTATGATTGACCGTCTGCGCCTCGTTCCGGCTAAGTAACAT GGAGCAGGTCGCGGATTTCGACACAATTTATCAGGCGATGATACAAATCTCCGTTGTACTT TGTTTCGCGCTTGGTATAATCGCTGGGGGTCAAAGATGAGTGTTTTAGTGTATTCTTTTGC CTCTTTCGTTTTAGGTTGGTGCCTTCGTAGTGGCATTACGTATTTTACCCGTTTAATGGAAA CTTCCTCATGAAAAAGTCTTTAGTCCTCAAAGCCTCTGTAGCCGTTGCTACCCTCGTTCCG ATGCTGTCTTTCGCTGCTGAGGGTGACGATCCCGCAAAAGCGGCCTTTAACTCCCTGCAAG CCTCAGCGACCGAATATATCGGTTATGCGTGGGCGATGGTTGTTGTCATTGTCGGCGCAAC TATCGGTATCAAGCTGTTTAAGAAATTCACCTCGAAAGCAAGCTGATAAACCGATACAAT TAAAGGCTCCTTTTGGAGCCTTTTTTTTGGAGATTTTCAACGTGAAAAAATTATTATTCGC AATTCCTTTAGTTGTTCCTTTCTATTCTCACTCCGCTGAAACTGTTGAAAGTTGTTTAGCAA AATCCCATACAGAAAATTCATTTACTAACGTCTGGAAAGACGACAAAACTTTAGATCGTT ACGCTAACTATGAGGGCTGTCTGTGGAATGCTACAGGCGTTGTAGTTTGTACTGGTGACG AAACTCAGTGTTACGGTACATGGGTTCCTATTGGGCTTGCTATCCCTGAAAATGAGGGTGG TGGCTCTGAGGGTGGCGGTTCTGAGGGTGGCGGTTCTGAGGGTGGCGGTACTAAACCTCC 
TGAGTACGGTGATACACCTATTCCGGGCTATACTTATATCAACCCTCTCGACGGCACTTAT CCGCCTGGTACTGAGCAAAACCCCGCTAATCCTAATCCTTCTCTTGAGGAGTCTCAGCCTC TTAATACTTTCATGTTTCAGAATAATAGGTTCCGAAATAGGCAGGGGGCATTAACTGTTTA TACGGGCACTGTTACTCAAGGCACTGACCCCGTTAAAACTTATTACCAGTACACTCCTGTA TCATCAAAAGCCATGTATGACGCTTACTGGAACGGTAAATTCAGAGACTGCGCTTTCCATT CTGGCTTTAATGAGGATTTATTTGTTTGTGAATATCAAGGCCAATCGTCTGACCTGCCTCA ACCTCCTGTCAATGCTGGCGGCGGCTCTGGTGGTGGTTCTGGTGGCGGCTCTGAGGGTGGT GGCTCTGAGGGTGGCGGTTCTGAGGGTGGCGGCTCTGAGGGAGGCGGTTCCGGTGGTGGC TCTGGTTCCGGTGATTTTGATTATGAAAAGATGGCAAACGCTAATAAGGGGGCTATGACC GAAAATGCCGATGAAAACGCGCTACAGTCTGACGCTAAAGGCAAACTTGATTCTGTCGCT ACTGATTACGGTGCTGCTATCGATGGTTTCATTGGTGACGTTTCCGGCCTTGCTAATGGTA ATGGTGCTACTGGTGATTTTGCTGGCTCTAATTCCCAAATGGCTCAAGTCGGTGACGGTGA TAATTCACCTTTAATGAATAATTTCCGTCAATATTTACCTTCCCTCCCTCAATCGGTTGAAT GTCGCCCTTTTGTCTTTGGCGCTGGTAAACCATATGAATTTTCTATTGATTGTGACAAAAT AAACTTATTCCGTGGTGTCTTTGCGTTTCTTTTATATGTTGCCACCTTTATGTATGTATTTTC TACGTTTGCTAACATACTGCGTAATAAGGAGTCTTAATCATGCCAGTTCTTTTGGGTATTC CGTTATTATTGCGTTTCCTCGGTTTCCTTCTGGTAACTTTGTTCGGCTATCTGCTTACTTTTC TTAAAAAGGGCTTCGGTAAGATAGCTATTGCTATTTCATTGTTTCTTGCTCTTATTATTGGG CTTAACTCAATTCTTGTGGGTTATCTCTCTGATATTAGCGCTCAATTACCCTCTGACTTTGT TCAGGGTGTTCAGTTAATTCTCCCGTCTAATGCGCTTCCCTGTTTTTATGTTATTCTCTCTGT AAAGGCTGCTATTTTCATTTTTGACGTTAAACAAAAAATCGTTTCTTATTTGGATTGGGAT AAATAATATGGCTGTTTATTTTGTAACTGGCAAATTAGGCTCTGGAAAGACGCTCGTTAGC GTTGGTAAGATTCAGGATAAAATTGTAGCTGGGTGCAAAATAGCAACTAATCTTGATTTA AGGCTTCAAAACCTCCCGCAAGTCGGGAGGTTCGCTAAAACGCCTCGCGTTCTTAGAATA CCGGATAAGCCTTCTATATCTGATTTGCTTGCTATTGGGCGCGGTAATGATTCCTACGATG AAAATAAAAACGGCTTGCTTGTTCTCGATGAGTGCGGTACTTGGTTTAATACCCGTTCTTG GAATGATAAGGAAAGACAGCCGATTATTGATTGGTTTCTACATGCTCGTAAATTAGGATG GGATATTATTTTTCTTGTTCAGGACTTATCTATTGTTGATAAACAGGCGCGTTCTGCATTAG CTGAACATGTTGTTTATTGTCGTCGTCTGGACAGAATTACTTTACCTTTTGTCGGTACTTTA TATTCTCTTATTACTGGCTCGAAAATGCCTCTGCCTAAATTACATGTTGGCGTTGTTAAATA TGGCGATTCTCAATTAAGCCCTACTGTTGAGCGTTGGCTTTATACTGGTAAGAATTTGTAT AACGCATATGATACTAAACAGGCTTTTTCTAGTAATTATGATTCCGGTGTTTATTCTTATTT AACGCCTTATTTATCACACGGTCGGTATTTCAAACCATTAAATTTAGGTCAGAAGATGAAA TTAACTAAAATATATTTGAAAAAGTTTTCTCGCGTTCTTTGTCTTGCGATTGGATTTGCATC AGCATTTACATATAGTTATATAACCCAACCTAAGCCGGAGGTTAAAAAGGTAGTCTCTCA GACCTATGATTTTGATAAATTCACTATTGACTCTTCTCAGCGTCTTAATCTAAGCTATCGCT ATGTTTTCAAGGATTCTAAGGGAAAATTAATTAATAGCGACGATTTACAGAAGCAAGGTT ATTCACTCACATATATTGATTTATGTACTGTTTCCATTAAAAAAGGTAATTCAAATGAAAT TGTTAAATGTAATTAATTTTGTTTTCTTGATGTTTGTTTCATCATCTTCTTTTGCTCAGGTAA TTGAAATGAATAATTCGCCTCTGCGCGATTTTGTAACTTGGTATTCAAAGCAATCAGGCGA ATCCGTTATTGTTTCTCCCGATGTAAAAGGTACTGTTACTGTATATTCATCTGACGTTAAAC CTGAAAATCTACGCAATTTCTTTATTTCTGTTTTACGTGCAAATAATTTTGATATGGTAGGT TCTAACCCTTCCATTATTCAGAAGTATAATCCAAACAATCAGGATTATATTGATGAATTGC CATCATCTGATAATCAGGAATATGATGATAATTCCGCTCCTTCTGGTGGTTTCTTTGTTCCG CAAAATGATAATGTTACTCAAACTTTTAAAATTAATAACGTTCGGGCAAAGGATTTAATA CGAGTTGTCGAATTGTTTGTAAAGTCTAATACTTCTAAATCCTCAAATGTATTATCTATTG ACGGCTCTAATCTATTAGTTGTTAGTGCTCCTAAAGATATTTTAGATAACCTTCCTCAATTC CTTTCAACTGTTGATTTGCCAACTGACCAGATATTGATTGAGGGTTTGATATTTGAGGTTC AGCAAGGTGATGCTTTAGATTTTTCATTTGCTGCTGGCTCTCAGCGTGGCACTGTTGCAGG CGGTGTTAATACTGACCGCCTCACCTCTGTTTTATCTTCTGCTGGTGGTTCGTTCGGTATTT TTAATGGCGATGTTTTAGGGCTATCAGTTCGCGCATTAAAGACTAATAGCCATTCAAAAAT 


\begin{tabular}{|l}
\hline ATTGTCTGTGCCACGTATTCTTACGCTTTCAGGTCAGAAGGGTTCTATCTCTGTTGGCCAG \\
AATGTCCCTTTATTACTGGTCGTGTGACTGGTGAATCTGCCAATGTAAATAATCCATTTC \\
AGACGATTGAGCGTCAAAATGTAGGTATTTCCATGAGCGTTTTCCTGTTGCAATGGCTGG \\
CGGTAATATTGTTCTGGATATTACCAGCAAGGCCGATAGTTTGAGTTCTTCTACTCAGGCA \\
AGTGATGTTATTACTAATCAAAGAAGTATTGCTACAACGGTTAATTTGCGTGATGGACAG \\
ACTCTTTACTCGGTGGCCTCACTGATTATAAAAACACTTCTCAGGATTCTGGCGTACCGT \\
TCCTGTCTAAAATCCCTTTAATCGGCCTCCTGTTTAGCTCCCGCTCTGATTCTAACGAGGA \\
AAGCACGTTATACGTGCTCGTCAAAGCAACCATAGTACGCGCCCTGTAGCGGCGCATTAA \\
GCGCGGCGGGTGTGGTGGTTACGCGCAGCGTGACCGCTACACTTGCCAGCGCCCTAGCGC \\
CCGCTCCTTTCGCTTTCTTCCCTTCCTTTCTCGCCACGTTCGCCGGCTTTCCCCGTCAAGCTC \\
TAAATCGGGGGCTCCCTTTAGGGTTCCGATTTAGTGCTTTACGGCACCTCGACCCCAAAAA \\
ACTTGATTTGGGTGATGGTTCACGTAGTGGGCCATCGCCCTGATAGACGGTTTTTCGCCCT \\
TTGACGTTGGAGTCCACGTTCTTTAATAGTGGACTCTTGTTCCAAACTGGAACAACACTCA \\
ACCCTATCTCGGGCTATTCTTTTGATTTATAAGGGATTTTGCCGATTTCGGAACCACCATC \\
AAACAGGATTTTCGCCTGCTGGGGCAAACCAGCGTGGACCGCTTGCTGCAACTCTCTCAG \\
GGCCAGGCGGTGAAGGGCAATCAGCTGTTGCCCGTCTCACTGGTGAAAAGAAAAACCACC \\
CTGGCGCCCAATACGCAAACCGCCTCTCCCCGCGCGTTGGCCGATTCATTAATGCAGCTGG \\
CACGACAGGTTTCCCGACTGGAAAGCGGGCAGTGAGCGCAACGCAATTAATGTGAGTTAG \\
CTCACTCATTAGGCACCCCAGGCTTTACACTTTATGCTTCCGGCTCGTATGTTGTGTGGAA \\
TTGTGAGCGGATAACAATTTCACACAGGAAACAGCTATGACCATGATTACGAATTCGAGC \\
TCGGTACCCGGGGATCCTCCGTCTTTATCGAGGTAACAAGCACCACGTAGCTTAAGCCCTG \\
TTTACTCATTACACCAACCAGGAGGTCAGAGTTCGGAGAAATGATTTATGTGAAATGCGT \\
CAGCCGATTCAAGGCCCCTATATTCGTGCCCACCGACGAGTTGCTTACAGATGGCAGGGC \\
CGCACTGTCGGTATCATAGAGTCACTCCAGGGCGAGCGTAAATAGATTAGAAGCGGGGTT \\
ATTTTGGCGGGACATTGTCATAAGGTTGACAATTCAGCACTAAGGACACTTAAGTCGTGC \\
GCATGAATTCACAACCACTTAGAAGAACATCCACCCTGGCTTCTCCTGAGAA
\end{tabular}


Table S3.

Origami staples. Hairpin binding sites are labeled in red, crosslinker sites are highlighted in gray.

To make the $\Delta 32$ structure, staples that are underlined were withheld from assembly solution.

\begin{tabular}{|c|c|c|c|}
\hline Start & End & Sequence & Length \\
\hline $0[71]$ & $5[71]$ & AATAGTGACAAGACAAAATTCTGTTCGAGCCA & 32 \\
\hline $0[103]$ & $5[103]$ & CGATAGCTATCGTCGCACAAAATCGAATTATT & 32 \\
\hline $0[135]$ & $5[135]$ & TTTGATTACACTTGCCGAATGGCTTGGCCAAC & 32 \\
\hline $0[167]$ & $5[167]$ & ATCACGCAGCCTTGCTGGTTAAGAAAGCGTTG & 32 \\
\hline $0[199]$ & $5[199]$ & AACCGTCTAGAGTCCAATTAATGATCTTTTCA & 32 \\
\hline $0[231]$ & $5[231]$ & ATATGATACTAGCTGAGAGTAACAGCATTAAA & 32 \\
\hline$\underline{\underline{0[263]}}$ & 5[263] & GAGAAAGGTATTTTTGGTAGCCAGTAACCAAT & $\underline{\underline{32}}$ \\
\hline $0[295]$ & $5[295]$ & TGAGTAATTCGGTTGTATTGCTGAGTAGCTCA & $\overline{32}$ \\
\hline $0[327]$ & $5[327]$ & TTAGAACCTACTTTTGAGAGGTCAGTACGGTG & 32 \\
\hline $0[359]$ & $5[359]$ & CCGATAGTATGACAACCTCATCTTAAATACGT & 32 \\
\hline $0[391]$ & $5[391]$ & CTTTCGAGATATTCGGAGGCGAAATAAAAGTT & 32 \\
\hline $0[423]$ & $5[423]$ & GGAGCCTTAGCGGAGTTGCTCAGTTGTATCAC & 32 \\
\hline $1[56]$ & $7[63]$ & TCCAATCGATTTATCAAATTTACG & 24 \\
\hline $1[88]$ & $7[95]$ & TTCTGTAATAGATTAAGAAACAAT & 24 \\
\hline $1[120]$ & $7[127]$ & CTTAACATGTAATAGACGCGAACT & 24 \\
\hline $1[152]$ & $7[159]$ & AACTATCGAATTAACCAAAATACC & 24 \\
\hline $1[184]$ & $7[191]$ & TTGGAACAATCACCGACACTGCCC & 24 \\
\hline $1[216]$ & $7[223]$ & ACTCCGTTTTCAACCACCGTGGGA & 24 \\
\hline$\underline{\underline{1}[248]}$ & $\underline{\underline{\underline{7}}[255]}$ & AGGGTAGCCCGGAGACGGATAGGT & $\underline{\underline{24}}$ \\
\hline $1[280]$ & $7[287]$ & AAGCTAAAGTGTAGGTTCAAAGCG & 24 \\
\hline $1[312]$ & $7[319]$ & CCCTGTAACTCATATACAGGTCAG & 24 \\
\hline $1[344]$ & $7[351]$ & TTCCGACATGCGCAACTATACCAA & 24 \\
\hline $1[376]$ & $7[383]$ & TAACCGATGTGAATTTTTTGTATC & 24 \\
\hline $1[408]$ & $7[415]$ & ACAGTTTCTAATTGTAGCCTATTT & 24 \\
\hline $2[71]$ & $4[64]$ & TATTTTAGTAGAAAAATATCATAT & 24 \\
\hline $2[103]$ & $4[96]$ & ATATGTGATTTCATTTATGATGAA & 24 \\
\hline $2[135]$ & $4[128]$ & CAGGAAAAAAATACCTGTCACACG & 24 \\
\hline $2[167]$ & $4[160]$ & ATCCAGAAAATGGTCCCAAGCGGA & 24 \\
\hline $2[199]$ & $4[192]$ & ATCAAAAGAAATCCTGCCCTTCAC & 24 \\
\hline $2[231]$ & $4[224]$ & GATGAACGAACTAGCAATTGTAAA & 24 \\
\hline $2[263]$ & $4[256]$ & TTGCCTGAATAATCAGAAAACAGG & $\underline{\underline{24}}$ \\
\hline $2[295]$ & $4[288]$ & AGCAATAACTATATTTGTTTGACC & 24 \\
\hline $2[327]$ & $4[320]$ & CATACAGGGTGGCATCTTGATTCC & 24 \\
\hline $2[359]$ & $4[352]$ & GGATCGTCGCGAAAGATTTCATGA & 24 \\
\hline $2[391]$ & $4[384]$ & AGGCTTGCGGCCATGTGAACCTAC & 24 \\
\hline $2[423]$ & $4[416]$ & GTAAATGAAGTACAAAACCCTCAT & 24 \\
\hline $3[48]$ & $5[63]$ & CCGGAATCATAATTACTTAATTTCAGGCATTT & 32 \\
\hline $3[80]$ & $5[95]$ & TAATTACATTTAACAAGTGAATAAAAGGAGGC & 32 \\
\hline $3[112]$ & $5[127]$ & TTTTTTAATGGCATGGACGCTAAAGGACATTC & 32 \\
\hline $3[144]$ & $5[159]$ & ACGCTCAATCGTCTGACAATATTATGACCTGA & 32 \\
\hline
\end{tabular}




\begin{tabular}{|c|c|c|c|}
\hline $3[176]$ & $5[191]$ & TTGCCCCAGCAGGCGAAATAGCCCGTGGTTTT & 32 \\
\hline $3[208]$ & $5[223]$ & TGGTTCCGAAACGTAAGTAATTCGTAAAATTC & 32 \\
\hline$\underline{\underline{3}[240]}$ & $5[255]$ & ATATGTACCCCGGTTGGAGTCTGGTCATTTTT & $\underline{\underline{32}}$ \\
\hline $3[272]$ & $5[287]$ & TCAATAACCTGTTTAGAGCCTCAGATAATGCT & 32 \\
\hline $3[304]$ & $5[319]$ & GGCGCGAGCTGAAAAGCAAGGCAACAACTAAA & 32 \\
\hline $3[336]$ & $5[351]$ & TAATAGTAGTACAGCAACCCTGCAAACGGGTA & 32 \\
\hline $3[368]$ & $5[383]$ & GAACGAGGGTAGCAACAGGGAGTTCACCAACC & 32 \\
\hline $3[400]$ & $5[415]$ & ACTGAGTTTCGTCACCATTTTCTGGGAATAGG & 32 \\
\hline $4[63]$ & $14[56]$ & GCGTTATAGCACCCAGCCGGTATTCGTCAAAAGACGGGAG & 40 \\
\hline $4[95]$ & $14[88]$ & ACAAACATAATTCATCTACCATATAGACATTAAGTAAATA & 40 \\
\hline $4[127]$ & $14[120]$ & ACCAGTAAATAATCAACCTCACTTATTAGAGCTTTGCCCG & 40 \\
\hline 4[159] & $14[152]$ & TTATTTACAGTTGAAAAGCATCACATTTAGAATTACAAAC & 40 \\
\hline $4[191]$ & $14[184]$ & CGCCTGGCCAGGGCTTCTCGAATTCTCGTCGGAGTGACTC & 40 \\
\hline $4[223]$ & 14[216] & CGTTAATAGATTGCGGATCGGAAACAGTGCCACCCCGCTT & 40 \\
\hline $4[255]$ & $14[248]$ & $\begin{array}{l}\text { AAGATTGTGCGAAAGGCCATTCAGGGATGTTCGTCAACCT } \\
\end{array}$ & 40 \\
\hline $4[287]$ & $14[280]$ & ATTAGATACCTCGTTTTATTCATTGCATAATATACGTCGA & 40 \\
\hline $4[319]$ & 14[312] & CAATTCTGTAGCGAGAGATAGCGTTTACAGGTTTATACCA & 40 \\
\hline $4[351]$ & 14[344] & GGAAGTTTAGATAGGCGCGCAGGGATAAGGCTAATTACCT & 40 \\
\hline $4[383]$ & 14[376] & AGAGGCTTCTTGACAAGAAAGAGGGAGTAGTAGAGATGGT & 40 \\
\hline $4[415]$ & 14[408] & TTTCAGGGCCACCCTCCGATTGGCGAATCAAGGTCACCAA & 40 \\
\hline $6[63]$ & $15[63]$ & CCAGACGAGGAATCATGCTGTCTTAAGAGCAA & 32 \\
\hline $6[87]$ & $0[72]$ & CGACAAAACATCGGGAGACGCTGAGAAGAGTC & 32 \\
\hline $6[95]$ & $15[95]$ & GCGCATACTATTTGCATATACAGTTCTTACCG & 32 \\
\hline 6[119] & $0[104]$ & TTATGAATTGCTTATGATCCTTGAAAACATAG & 32 \\
\hline $6[127]$ & $15[127]$ & ATTAGTCTCGTGTATTGGTCAAGAAGATAGCC & 32 \\
\hline $6[151]$ & $0[136]$ & CACAGACACGCCATTAGTTGTAGCAATACTTC & 32 \\
\hline 6[159] & $15[159]$ & ATACGTGGCTGAGAGCACCACCAGGAGGAAAC & 32 \\
\hline $6[183]$ & $0[168]$ & ACGCGCGGGTTGCGCTGTAAAAGAGTCTGTCC & 32 \\
\hline 6[191] & 15[191] & ATCGGCCAGTGTGAAAAGCCTGGGGAACTGGC & 32 \\
\hline $6[215]$ & $0[200]$ & GATTCGTGACCTGTCTACGTCAAAGGGCGAAA & 32 \\
\hline $6[223]$ & $15[223]$ & ACCCGTCGCAAATCGGACAGTCATGTATGTTA & 32 \\
\hline$\underline{\underline{6[247]}}$ & $\underline{\underline{0[232]}}$ & AACATTAACCGTAATGAGTCAAATCACCATCA & $\underline{\underline{32}}$ \\
\hline $6[255]$ & $15[255]$ & CTTTCATCAGCTTTCCAACCGTGCAAGGTGGC & $\underline{\underline{32}}$ \\
\hline 6[279] & $0[264]$ & CGCGTCTGTTCGAGCTAAAGATTCAAAAGGGT & 32 \\
\hline $6[287]$ & $15[287]$ & ATATAATTCCCTCAAAGACTTCAAACCACGGA & 32 \\
\hline $6[311]$ & $0[296]$ & GATGGCTTAACTCCAATTTTAAATGCAATGCC & 32 \\
\hline 6[319] & $15[319]$ & TTTTTGCGGAATGACCTTGCATCATAGAAAAT & 32 \\
\hline $6[343]$ & $0[328]$ & AGCGCTCCTAATTGATGCAAGGATAAAAATTT & 32 \\
\hline $6[351]$ & $15[351]$ & TGACCCCCCCTTACTTCATGTGACACAAAAGG & 32 \\
\hline $6[375]$ & $0[360]$ & AGAATACAAACGGAGACTTAAACAGCTTGATA & 32 \\
\hline $6[383]$ & 15 [383] & GAGGCAAAAATCATAATGATAAATGGAAGGTA & 32 \\
\hline $6[407]$ & $0[392]$ & GATAAGTGTGCCCCCTTCGGTTTATCAGCTTG & 32 \\
\hline $6[415]$ & 15 [415] & ACCAGGCGTCATTAAAGGGGTCAGAAGGTGAA & 32 \\
\hline
\end{tabular}




\begin{tabular}{|c|c|c|c|}
\hline $6[439]$ & $0[424]$ & AGGATTAGGAAACATGAAAAAAGGCTCCAAAA & 32 \\
\hline $7[64]$ & $13[71]$ & AGCATGTAAATAATCGTACCGCGCGCGCATTAATGAAAAT & 40 \\
\hline $7[96]$ & $13[103]$ & AACGGATTCAGATGAACGTAAAACAAAGTTTGTCATTTTG & 40 \\
\hline $7[128]$ & $13[135]$ & GATAGCCCGGTGAGGCAACACCGCTTAAATCCCGTCAATA & 40 \\
\hline $7[160]$ & $13[167]$ & GAATCACACTAACCGACAGCAGCACGGAGACTGTATTCCC & 40 \\
\hline $7[192]$ & $13[199]$ & GCTTTCCAAAGTGTAATTGTTATCCGCCCTGGTGGGCACG & 40 \\
\hline $7[224]$ & $13[231]$ & ACAAACGGGGACGACGCCTCAGGACAAAATAAAGCTTTCT & 40 \\
\hline 7[256] & $13[263]$ & CACGTTGGCGCATCGTGGCACCGCGCTGAATTTTCTAAGT & $\underline{\underline{40}}$ \\
\hline $7[288]$ & $13[295]$ & AACCAGACGCCCGAAATGCTTTAAGAAAAATCAAACGAAC & 40 \\
\hline $7[320]$ & $13[327]$ & GATTAGAGAAAGCGGAATAAATCACTGGCTCAAGAAAGAT & 40 \\
\hline $7[352]$ & $13[359]$ & GCGCGAAAACCTGCTCAGCCGGAATCATTGTGTGCCCTGA & 40 \\
\hline $7[384]$ & 13 [391] & ATCTATAAGCCCGGCCGGGAACCGAGCGGCTTAATTGACC & 40 \\
\hline $7[416]$ & $13[423]$ & CGGAACCTGTTTTAACGCCAGAATCCGGAAACTTTGCCTT & 40 \\
\hline $8[79]$ & $2[72]$ & CTTCAATCGAAACTTAGGTAAAGTAGAACGCGTTCAAATA & 40 \\
\hline $8[111]$ & $2[104]$ & TTTAACGTCGCCTGATACCAAGTTTATTAATTAAATCAAT & 40 \\
\hline $8[143]$ & $2[136]$ & AAAACAGATAAAACATATATTTTTTGAGTAGACATTGCAA & 40 \\
\hline $8[175]$ & $2[168]$ & TGAGTGAGTTAATTGCGGAGAGGCGGTGTGTTGTTGAAAT & 40 \\
\hline $8[207]$ & $2[200]$ & GAAGCATAGTCGGGAACCAGCTGCCTATTAAACCTTATAA & 40 \\
\hline$\underline{\underline{8}[239]}$ & $\underline{\underline{2}[232]}$ & GTTTGAGGCGGATTGAATGTGAGCTAAATTAAAGAGAATC & $\underline{\underline{40}}$ \\
\hline $8[271]$ & $2[264]$ & TTTATGGGTGTAGTAAGCCTTCCTAGAGATCTTCAGGTCA & 40 \\
\hline $8[303]$ & $2[296]$ & AAGAGGAACGGAAGCAAGAGCTTAACCAAAAACAAAATTA & 40 \\
\hline $8[335]$ & $2[328]$ & TCAGAAGCAGTACCTTTTTTGATACGGGAGAACAATAAAT & 40 \\
\hline $8[367]$ & $2[360]$ & AATCCGCGCAAAGTACCTAAAACAAACCATCGCTTTTGCG & 40 \\
\hline $8[399]$ & $2[392]$ & GTAACAGTACAGTTAACCGTCGAGTCGTAAACTTTGCCTG & 40 \\
\hline $8[431]$ & $2[424]$ & GGTAATAAATTATTCTCGGGGTTTGAGAATAGAGACGTTA & 40 \\
\hline $10[71]$ & $12[56]$ & AGGCTTATCTACAATTTCTTACCAACGCTAAC & 32 \\
\hline $10[103]$ & $12[88]$ & TTAGAACCAATATAATCGGAATTATCATCATA & 32 \\
\hline $10[135]$ & $12[120]$ & TATCAAACTATCTGGTTAGGAGCACTAACAAC & 32 \\
\hline $10[167]$ & $12[152]$ & AAATCTAAGGACTGGTACCTCATTGAGGAAGG & 32 \\
\hline $10[199]$ & $12[184]$ & GTACCGAGAAGCTACGGCATTTCACATAAATC & 32 \\
\hline $10[231]$ & $12[216]$ & GAAGGGCGGCCTCTTCCACGACGTTGTAAAAC & 32 \\
\hline$\underline{\underline{10}[263]}$ & $\underline{\underline{12[248]}}$ & GCCATTCGGGGATGTGATTAAGTTGGGTAACG & $\underline{\underline{32}}$ \\
\hline $10[295]$ & $12[280]$ & GTCATAAAACCAGACGGGAATTACGAGGCATA & 32 \\
\hline $10[327]$ & $12[312]$ & TTAGACTGGGCTTTTGCATTCAACTAATGCAG & 32 \\
\hline $10[359]$ & $12[344]$ & CAGACCAGTGGCTGACAACAAAGCTGCTCATT & 32 \\
\hline $10[391]$ & $12[376]$ & CCAACTTTGAAGAACCCACCGCCGGATATTCA & 32 \\
\hline $10[423]$ & $12[408]$ & AGGTCAGAAGAACCGCTTCATAATCAAAATCA & 32 \\
\hline $10[79]$ & $3[79]$ & GATATAGAGTAATAAGCAATTTAGGCCTGAAT & 32 \\
\hline $10[143]$ & $3[143]$ & ACCTCAAAAGAGATAGATTCACCAACATTTTG & 32 \\
\hline $10[111]$ & $3[111]$ & TGGAAGGGCATTTCAAAAAAGAAGGAATTACC & 32 \\
\hline $10[175]$ & $3[175]$ & TGGTCGAACGTATTGGAGTTGCAGACGCTGGT & 32 \\
\hline $10[207]$ & $3[207]$ & ATCCCCGGCCAGTGAGGCTGATTGTTTGATGG & 32 \\
\hline$\underline{\underline{10[239]}}$ & $\underline{\underline{3[239]}}$ & ACTGTTGGTTTTTGTTATATTTAATGTCAATC & $\underline{\underline{\underline{32}}}$ \\
\hline
\end{tabular}




\begin{tabular}{|c|c|c|c|}
\hline $10[271]$ & $3[271]$ & GGCAAAGCAGGAACGCAAACCCCAAAAAGTGG & 32 \\
\hline $10[303]$ & $3[303]$ & GCGGAATCACATGTTTTAGATTTATCATTTGG & 32 \\
\hline $10[335]$ & $3[335]$ & TAAAATGTTCTGGAAGTATAACAGAATTCTAC & 32 \\
\hline $10[367]$ & $3[367]$ & ACGGTGTAAATGCCACAAAGACTTCAGCATCG & 32 \\
\hline 10 [399] & $3[399]$ & TCACATGAGATATAAGCCCAATAGACCGTAAC & 32 \\
\hline $10[431]$ & $3[431]$ & GTTGAGGCCGTACTCAGAGCCACCCTACAACG & 32 \\
\hline $11[80]$ & $1[87]$ & ATGATGGCCAAGAAAAAGAATATACCTACTTTAGAAATGC & 40 \\
\hline $11[112]$ & $1[119]$ & TTTGGATTTAATGAGCTTACCAAGCAGTACATAATTTTCC & 40 \\
\hline $11[144]$ & $1[151]$ & AAATCAACATTGGCAGAACCCTTCCCGCCAGCAGAACTCA & 40 \\
\hline $11[176]$ & $1[183]$ & TGAGTAAACCTGAGAGGCGCCAGGGAGATAGGGTTCCAGT & 40 \\
\hline $11[208]$ & $1[215]$ & GTTACCTCTTTCAACAACGGGTGTGCAAAATCGAACGTGG & 40 \\
\hline $11[240]$ & $1[247]$ & GCCAGCTGATAAGCAAAAATCAGCAGCAAACATGCCGGAG & $\underline{\underline{40}}$ \\
\hline $11[272]$ & $1[279]$ & ATCATAACCATTTCGCCATCAAAAAGCGGCTAACAAAATA & 40 \\
\hline $11[304]$ & $1[311]$ & AACCAAAACGAACGAGTAAATATGAGAATTAGCATTATGA & 40 \\
\hline $11[336]$ & $1[343]$ & GTTTTGCCCCATTCCATTTCATTATTAACATCGCCTTTAT & 40 \\
\hline $11[368]$ & $1[375]$ & AGAGTAATTGAGGACTTACGAAGGAAAGGCCGCCCACGCA & 40 \\
\hline $11[400]$ & $1[407]$ & CAGAGCCGATAGCAAGTATAGCCCTATGGGATAACTTTCA & 40 \\
\hline $11[432]$ & $1[439]$ & GAGCCACCCACCCTCAGGAGGTTTGTCTTTCCAAAGGAAC & 40 \\
\hline 12[55] & 9[55] & GAGCGTCTTTGTTTAACTAAGAACTCATCGTA & 32 \\
\hline 12[87] & 9[87] & TTCCTGATTTACAGAGCAAAATCAAAGCAAAT & 32 \\
\hline 12[119] & $9[119]$ & TAACCACCAGAAATAGCTGAATAAAGAAATTG & 32 \\
\hline 12 [151] & $9[151]$ & TTATCTAAATTTGAGGCTTGCTGAGTGCCACG & 32 \\
\hline $12[183]$ & $9[183]$ & ATTTCTCCGTAAGCAACGTAATCATGTTTCCT & 32 \\
\hline $12[215]$ & $9[215]$ & GACTGAATGGCCTGGCGACGGAGGATTCCACA & 32 \\
\hline 12[247] & $9[247]$ & CCAGGGTTGCCAGGGTGCTGCGCACTCCAGCC & 32 \\
\hline $12[279]$ & $9[279]$ & GTAAGAGCATTCATGCGAAAACCACCGGATCC & 32 \\
\hline $12[311]$ & $9[311]$ & ATACATAACAACATTACCAATACTGAAAACGA & 32 \\
\hline 12 [343] & $9[343]$ & CAGTTTAGTGAGATGAGGTAATAGGTCTTTAC & 32 \\
\hline $12[375]$ & $9[375]$ & TTACCCAAACCAGAACACAGATGAAGACGGTC & 32 \\
\hline 12 [407] & $9[407]$ & CCGGAACCTAGCGACACTTGATATATAAATCC & 32 \\
\hline $13[72]$ & 11[79] & AGCAGCCTTATCTGAATTATCCAG & 24 \\
\hline $13[104]$ & $11[111]$ & CGGAACAAAGAAGGAGCCTGATTG & 24 \\
\hline $13[136]$ & $11[143]$ & GATAATACAATATCTTCAGTTGGC & 24 \\
\hline $13[168]$ & $11[175]$ & TGCCATCTGAACTCTGTGGTGTAA & 24 \\
\hline $13[200]$ & $11[207]$ & AATATAGGCGGCTGACTGGTGCTT & 24 \\
\hline $13[232]$ & $\underline{11[239]}$ & CAGGAGAATTCCCAGTGCTATTAC & $\underline{\underline{\underline{2}}}$ \\
\hline $13[264]$ & $11[271]$ & GGTTGTGAAACAGGCGCTGCAACT & 24 \\
\hline $13[296]$ & $11[303]$ & TAACGGAACGCCAAAAACGATAAA & 24 \\
\hline $13[328]$ & $11[335]$ & TCATCAGTGAATACCACAAAAGAA & 24 \\
\hline $13[360]$ & $11[367]$ & CGAGAAACATCAACGTCTTCATCA & 24 \\
\hline $13[392]$ & $11[399]$ & GTAATCAGAGAGCCACGCCTCCCT & 24 \\
\hline 13[424] & $11[431]$ & TAGCGTCAGCCATCTTCACCCTCA & 24 \\
\hline $14[55]$ & $8[47]$ & ААТТААСТССААТААТТССТТАТСА & 25 \\
\hline
\end{tabular}




\begin{tabular}{|l|l|l|l|}
\hline $14[87]$ & $8[80]$ & ACATAAAAAATAGCTAAACAGTAC & 24 \\
\hline $14[119]$ & $8[112]$ & AACGTTATAAGTAAGCTTTTCAGG & 24 \\
\hline $14[151]$ & $8[144]$ & AATTCGACAGGAAACCCAGAAGAT & 24 \\
\hline $14[183]$ & $8[176]$ & TATGATACACCCAAAAGTGCCTAA & 24 \\
\hline $14[215]$ & $8[208]$ & CTAATCTAATTACGCAACGAGCCG & 24 \\
\hline$\underline{14[247]}$ & $\underline{8[240]}$ & TATGACAACATACATAATCTGCCA & $\underline{24}$ \\
\hline $14[279]$ & $8[272]$ & CTTAAGTGGCAAAGACATATCGCG & 24 \\
\hline $14[311]$ & $8[304]$ & GTCAGGACACAATCAAAAAAGATT & 24 \\
\hline $14[343]$ & $8[336]$ & TATGCGATCGCCAAAGTATTATAG & 24 \\
\hline $14[375]$ & $8[368]$ & TTAATTTCTGAGGGAGTGTGTCGA & 24 \\
\hline $14[407]$ & $8[400]$ & TGAAACCAATTCATTATGCCTTGA & 24 \\
\hline $15[64]$ & $9[79]$ & GAAACAATGAAATAGCACAGGGAACCAATAGC & 32 \\
\hline $15[96]$ & $9[111]$ & AAGCCCTTTTTAAGAATAATTTTAAGAAATAA & 32 \\
\hline $15[128]$ & $9[143]$ & GAACAAAGTTACCAGAAACTCGTACTGCAACA & 32 \\
\hline $15[160]$ & $9[175]$ & GCAATAATAACGGAATCGACAGTGAATATAGC & 32 \\
\hline $15[192]$ & $9[207]$ & ATGATTAAGACTCCTTTTTACGCTCGCTCACA & 32 \\
\hline $15[224]$ & $9[239]$ & GCAAACGTAGAAAATATGTCCCGCAGATCGCA & 32 \\
\hline$\underline{15[256]}$ & $\underline{9[271]}$ & AACATATAAAAGAAACTCCTTAGTTTCTGGTG & $\underline{32}$ \\
\hline $15[288]$ & $9[303]$ & ATAAGTTTATTTTGTCGTTGGGAAACAGTTCA & 32 \\
\hline $15[320]$ & $9[335]$ & TCATATGGTTTACCAGTTTAAGAAAAAATCAG & 32 \\
\hline $15[352]$ & $9[367]$ & GCGACATTCAACCGATAACTTTAACGAGGCGC & 32 \\
\hline $15[384]$ & $9[399]$ & AATATTGACGGAAATTTCGATAGCAACAACAA & 32 \\
\hline $15[416]$ & $9[439]$ & TTATCACCGTCACCGATAGCAAGGGGAAAGCGCAGTCTCT & 40 \\
\hline
\end{tabular}


Table S4.

Reported volumetric phase transition dynamics of thermo-responsive polymer particles.

\begin{tabular}{|c|c|c|c|c|}
\hline Reference & Year & Particle size & Characterization & Dynamics \\
\hline Tanaka et $\mathrm{al}^{26}$ & 1988 & $\begin{array}{l}234 \mu \mathrm{m}-3.1 \text { time } \\
\text { smaller }\end{array}$ & water exchange heating & $\begin{array}{l}\text { The switching time of the temperature of } \\
\text { the water was about } 10 \mathrm{~s} \text {, which is much } \\
\text { shorter than the swelling or shrinking time } \\
\text { of most of the gels. }\end{array}$ \\
\hline $\begin{array}{l}\text { Andrew Lyon } \\
\text { Mostafa A. El- } \\
\text { Sayed }^{27}\end{array}$ & 2001 & $200 \mathrm{~nm}$ & $\begin{array}{l}\text { turbidity, laser T-jump } \\
\text { transmittance signal }\end{array}$ & ns to $\mu \mathrm{s}, 0.39 \mu \mathrm{s}$ \\
\hline Asher et $\mathrm{al}^{28}$ & 2004 & $\begin{array}{l}\text { self-assembling } \\
\text { crystalline } \\
\text { colloidal arrays, } \\
350 \mathrm{~nm}-125 \mathrm{~nm}\end{array}$ & $\begin{array}{l}\text { T-jump. Diffraction for } \\
\text { CCA } \\
\text { single particle: turbidity }\end{array}$ & $\begin{array}{l}\text { The shortest time } \sim 900 \text { ns kinetics } \\
\text { accounts for } \sim 25 \% \text { of the transmission } \\
\text { change, while the longer time } \sim \mathbf{2 0 \mu} \text { s } \\
\text { kinetics accounts for another } \sim 25 \% \text {. The } \\
\text { longest } \sim \mathbf{1 4 0 \mu} \text { s kinetics accounts for the } \\
\text { remaining } 50 \% \text { extinction increase. } \\
\text { Individual NIPAM sphere switching } \\
\text { occurs in the } \sim \mathbf{1 0 0} \text { ns time regime. }\end{array}$ \\
\hline Asher et $\mathrm{al}^{29}$ & 2009 & $160 \mathrm{~nm}-80 \mathrm{~nm}$ & $\begin{array}{l}\mathrm{T} \text {-jump and UV } \\
\text { resonance Raman } \\
\text { (UVRR) spectroscopy }\end{array}$ & $\begin{array}{l}\text { Mono-exponential collapse with } \boldsymbol{\tau} \sim \mathbf{3 6 0} \pm \\
\mathbf{8 5} \mathbf{~ n s}\end{array}$ \\
\hline Asher et $\mathrm{al}^{30}$ & 2018 & $404 \mathrm{~nm}-143 \mathrm{~nm}$ & $\begin{array}{l}\text { visible non-resonance } \\
\text { Raman T-jump }\end{array}$ & $\begin{array}{l}\text { The PNIPAM hydrophobic isopropyl and } \\
\text { methylene groups dehydrate with time } \\
\text { constants of } 109 \pm 64 \text { and } 104 \pm 44 \mathrm{ns,} \\
\text { initiating the volume collapse of } \\
\text { PNIPAM. The subsequent dehydration of } \\
\text { the PNIPAM amide groups is significantly } \\
\text { slower, as our group previously } \\
\text { discovered }(360 \pm 85 \mathrm{~ns})\end{array}$ \\
\hline $\mathrm{Hu}$ et $\mathrm{al}^{31}$ & 2009 & $60 \mu \mathrm{m}$ & $\begin{array}{l}\text { modeling and taking } \\
\text { image at different } \\
\text { timepoints }\end{array}$ & $10-100 \mathrm{sec}$ \\
\hline Marquez et al ${ }^{32}$ & 2006 & $\mu \mathrm{m}$ scale minigel & $\begin{array}{l}\text { modeling and taking } \\
\text { image at different } \\
\text { timepoints }\end{array}$ & $\sim$ sec scale \\
\hline Salaita et al ${ }^{33}$ & 2019 & $550 \mathrm{~nm}-350 \mathrm{~nm}$ & IR T-jump & $\begin{array}{l}\mathbf{1 . 4 2} \boldsymbol{\mu s}(40.7 \%), \mathbf{1 0 . 8 0} \boldsymbol{\mu s}(36.7 \%), \mathbf{1 4 2} \boldsymbol{\mu s} \\
(22.6 \%), 35^{\circ} \mathrm{C} \text { to } 45^{\circ} \mathrm{C}\end{array}$ \\
\hline
\end{tabular}


Table S5.

Single-exponential fits of T-jump \& mechanical unfolding kinetics of DNA hairpin

\begin{tabular}{|c|c|c|c|c|}
\hline \multicolumn{5}{|c|}{$\mathrm{T}$ jump } \\
\hline & & Replicate \#1 & Replicate \#2 & Replicate \#3 \\
\hline \multirow{3}{*}{$50^{\circ} \mathrm{C}$} & y0 & 1.04206 & 1.04874 & 1.09249 \\
\hline & A1 & -0.02576 & -0.03779 & -0.07818 \\
\hline & $\mathrm{t} 1$ & $-7.36 \mathrm{E}-05$ & $-4.17 \mathrm{E}-05$ & $-5.45 \mathrm{E}-05$ \\
\hline \multirow{3}{*}{$55^{\circ} \mathrm{C}$} & $\mathrm{y} 0$ & 1.11706 & 1.10222 & 1.10472 \\
\hline & $\mathrm{A} 1$ & -0.11873 & -0.09197 & -0.0954 \\
\hline & $\mathrm{t} 1$ & $-5.12 \mathrm{E}-05$ & $-3.55 \mathrm{E}-05$ & $-4.92 \mathrm{E}-05$ \\
\hline \multirow{3}{*}{$60^{\circ} \mathrm{C}$} & $\mathrm{y} 0$ & 1.25246 & 1.18715 & 1.14314 \\
\hline & A1 & -0.27482 & -0.18935 & -0.1496 \\
\hline & $\mathrm{t} 1$ & $-3.85 \mathrm{E}-05$ & $-3.18 \mathrm{E}-05$ & $-2.98 \mathrm{E}-05$ \\
\hline \multirow{3}{*}{$65^{\circ} \mathrm{C}$} & $\mathrm{y} 0$ & 1.27531 & 1.27014 & 1.11876 \\
\hline & A1 & -0.30072 & -0.29085 & -0.14145 \\
\hline & $\mathrm{t} 1$ & $-2.27 \mathrm{E}-05$ & $-2.10 \mathrm{E}-05$ & $-1.46 \mathrm{E}-05$ \\
\hline \multicolumn{5}{|c|}{ Force jump } \\
\hline & & Replicate \#1 & Replicate \#2 & Replicate \#3 \\
\hline \multirow{3}{*}{$45^{\circ} \mathrm{C}$} & $\mathrm{y} 0$ & 1.12115 & 1.08967 & 1.07291 \\
\hline & A1 & -0.111 & -0.06864 & -0.05888 \\
\hline & $\mathrm{t} 1$ & $-1.66 \mathrm{E}-04$ & $-3.70 \mathrm{E}-04$ & $-2.06 \mathrm{E}-04$ \\
\hline \multirow{3}{*}{$50^{\circ} \mathrm{C}$} & y0 & 1.20089 & 1.10659 & 1.08534 \\
\hline & A1 & -0.19963 & -0.09198 & -0.06815 \\
\hline & $\mathrm{t} 1$ & $-1.08 \mathrm{E}-04$ & $-1.66 \mathrm{E}-04$ & $-1.64 \mathrm{E}-04$ \\
\hline \multirow{3}{*}{$55^{\circ} \mathrm{C}$} & $\mathrm{y} 0$ & 1.29485 & 1.11821 & 1.05727 \\
\hline & $\mathrm{A} 1$ & -0.29847 & -0.11953 & -0.04222 \\
\hline & $\mathrm{t} 1$ & $-5.41 \mathrm{E}-05$ & $-6.72 \mathrm{E}-05$ & $-7.27 \mathrm{E}-05$ \\
\hline \multirow{3}{*}{$60^{\circ} \mathrm{C}$} & y0 & 1.23931 & 1.161 & 1.04781 \\
\hline & A1 & -0.24386 & -0.1643 & -0.03935 \\
\hline & $\mathrm{t} 1$ & $-2.91 \mathrm{E}-05$ & $-4.52 \mathrm{E}-05$ & $-5.07 \mathrm{E}-05$ \\
\hline \multirow{3}{*}{$65^{\circ} \mathrm{C}$} & y0 & 1.22235 & 1.18765 & 1.05924 \\
\hline & $\mathrm{A} 1$ & -0.2463 & -0.21118 & -0.0465 \\
\hline & $\mathrm{t} 1$ & $-1.88 \mathrm{E}-05$ & $-2.19 \mathrm{E}-05$ & $-2.59 \mathrm{E}-05$ \\
\hline
\end{tabular}


Table S6.

Statistical two-way ANOVA test of angle and force of the OPFC at different configuration and temperature.

\begin{tabular}{|c|c|c|c|c|c|c|}
\hline \multirow[b]{2}{*}{$\begin{array}{l}\text { Configuration- } \\
\text { Temperature) }\end{array}$} & \multicolumn{3}{|c|}{ Angle of bending } & \multicolumn{3}{|c|}{ Force } \\
\hline & Significant? & Summary & Adjusted P Value & Significant? & Summary & Adjusted P Value \\
\hline $\begin{array}{c}\mathrm{L}_{10 \mathrm{~nm}}-40^{\circ} \mathrm{C} \text { vs. } \\
\mathrm{L}_{10 \mathrm{~nm}}-55^{\circ} \mathrm{C}\end{array}$ & Yes & $* \star \star *$ & $<0.000000000000001$ & No & ns & 0.999999999 \\
\hline $\begin{array}{c}\mathrm{L}_{30 \mathrm{~nm}^{-}}-40^{\circ} \mathrm{C} \text { vs. } \\
\mathrm{L}_{30 \mathrm{~nm}}-55^{\circ} \mathrm{C}\end{array}$ & Yes & $* * * *$ & $<0.000000000000001$ & No & ns & 1 \\
\hline $\begin{array}{c}\mathrm{L}_{50 \mathrm{~nm}}-40^{\circ} \mathrm{C} \text { vs. } \\
\mathrm{L}_{50 \mathrm{~nm}}-55^{\circ} \mathrm{C}\end{array}$ & Yes & $* * * *$ & $<0.000000000000001$ & No & ns & >0.999999999999 \\
\hline $\begin{array}{c}\mathrm{L}_{70 \mathrm{~nm}}-40^{\circ} \mathrm{C} \text { vs. } \\
\mathrm{L}_{70 \mathrm{~nm}}-55^{\circ} \mathrm{C}\end{array}$ & Yes & $* * * *$ & $<0.000000000000001$ & No & ns & 0.999996866 \\
\hline $\begin{array}{l}\mathrm{L}_{90 \mathrm{~nm}}-40^{\circ} \mathrm{C} \text { vs. } \\
\mathrm{L}_{90 \mathrm{~nm}}-55^{\circ} \mathrm{C}\end{array}$ & Yes & $* * * *$ & $<0.000000000000001$ & No & ns & 0.791015348 \\
\hline $\begin{array}{l}\mathrm{L}_{110 \mathrm{~nm}}-40^{\circ} \mathrm{C} \text { vs. } \\
\mathrm{L}_{110 \mathrm{~nm}}-55^{\circ} \mathrm{C}\end{array}$ & Yes & $* * * *$ & $<0.000000000000001$ & No & ns & 0.168494339 \\
\hline $\begin{array}{c}\mathrm{L}_{110 \mathrm{~nm}}(\Delta 32)-40^{\circ} \mathrm{C} \\
\text { vs. } \mathrm{L}_{110 \mathrm{~nm}}(\Delta 32)- \\
55^{\circ} \mathrm{C}\end{array}$ & Yes & $* * * *$ & $<0.000000000000001$ & No & ns & 0.997914188 \\
\hline $\begin{array}{c}\mathrm{L}_{10 \mathrm{~nm}}-40^{\circ} \mathrm{C} \text { vs. } \\
\mathrm{L}_{30 \mathrm{~nm}}-40^{\circ} \mathrm{C}\end{array}$ & No & ns & 0.316370815 & No & ns & $>0.999999999999$ \\
\hline $\begin{array}{c}\mathrm{L}_{10 \mathrm{~nm}}-40^{\circ} \mathrm{C} \text { vs. } \\
\mathrm{L}_{50 \mathrm{~nm}}-40^{\circ} \mathrm{C}\end{array}$ & Yes & $* * * *$ & $<0.000000000000001$ & No & ns & $>0.999999999999$ \\
\hline $\begin{array}{c}\mathrm{L}_{10 \mathrm{~nm}}-40^{\circ} \mathrm{C} \text { vs. } \\
\mathrm{L}_{70 \mathrm{~nm}}-40^{\circ} \mathrm{C}\end{array}$ & Yes & $* * * *$ & $<0.000000000000001$ & No & ns & $>0.999999999999$ \\
\hline $\begin{array}{l}\mathrm{L}_{10 \mathrm{~nm}}-40^{\circ} \mathrm{C} \text { vs. } \\
\mathrm{L}_{90 \mathrm{~nm}}-40^{\circ} \mathrm{C}\end{array}$ & Yes & $* * * *$ & $<0.000000000000001$ & No & ns & $>0.999999999999$ \\
\hline $\begin{array}{c}\mathrm{L}_{10 \mathrm{~nm}}-40^{\circ} \mathrm{C} \text { vs. } \\
\mathrm{L}_{110 \mathrm{~nm}}-40^{\circ} \mathrm{C}\end{array}$ & Yes & $* * * *$ & 1.40249E-06 & No & ns & 0.999999996 \\
\hline $\begin{array}{c}\mathrm{L}_{10 \mathrm{~nm}}-40^{\circ} \mathrm{C} \text { vs. } \\
\mathrm{L}_{110 \mathrm{~nm}}(\Delta 32)-40^{\circ} \mathrm{C}\end{array}$ & Yes & **** & $<0.000000000000001$ & No & ns & $>0.999999999999$ \\
\hline $\begin{array}{c}\mathrm{L}_{10 \mathrm{~nm}}-55^{\circ} \mathrm{C} \text { vs. } \\
\mathrm{L}_{30 \mathrm{~nm}}-55^{\circ} \mathrm{C}\end{array}$ & Yes & $* * * *$ & $<0.000000000000001$ & No & ns & $>0.999999999999$ \\
\hline $\begin{array}{c}\mathrm{L}_{10 \mathrm{~nm}}-55^{\circ} \mathrm{C} \text { vs. } \\
\mathrm{L}_{50 \mathrm{~nm}}-55^{\circ} \mathrm{C} \\
\end{array}$ & Yes & $* * \star *$ & $<0.000000000000001$ & No & ns & 0.999999982 \\
\hline $\begin{array}{c}\mathrm{L}_{10 \mathrm{~nm}}-55^{\circ} \mathrm{C} \text { vs. } \\
\mathrm{L}_{70 \mathrm{~nm}}-55^{\circ} \mathrm{C}\end{array}$ & Yes & $* * * *$ & $<0.000000000000001$ & No & ns & 0.99937119 \\
\hline $\begin{array}{c}\mathrm{L}_{10 \mathrm{~nm}}-55^{\circ} \mathrm{C} \text { vs. } \\
\mathrm{L}_{90 \mathrm{~nm}}-55^{\circ} \mathrm{C}\end{array}$ & Yes & $* * * *$ & $<0.000000000000001$ & No & ns & 0.539003525 \\
\hline $\begin{array}{c}\mathrm{L}_{10 \mathrm{~nm}}-55^{\circ} \mathrm{C} \text { vs. } \\
\mathrm{L}_{110 \mathrm{~nm}}-55^{\circ} \mathrm{C}\end{array}$ & Yes & $* * * *$ & $<0.000000000000001$ & Yes & * & 0.029530476 \\
\hline $\begin{array}{c}\mathrm{L}_{10 \mathrm{~nm}}-55^{\circ} \mathrm{C} \text { vs. } \\
\mathrm{L}_{110 \mathrm{~nm}}(\Delta 32)-55^{\circ} \mathrm{C}\end{array}$ & Yes & $* * * *$ & $<0.000000000000001$ & No & ns & 0.983240496 \\
\hline $\begin{array}{c}\mathrm{L}_{30 \mathrm{~nm}}-40^{\circ} \mathrm{C} \text { vs. } \\
\mathrm{L}_{50 \mathrm{~nm}}-40^{\circ} \mathrm{C}\end{array}$ & Yes & $* * * *$ & $<0.000000000000001$ & No & ns & $>0.999999999999$ \\
\hline $\begin{array}{c}\mathrm{L}_{30 \mathrm{~nm}}-40^{\circ} \mathrm{C} \text { vs. } \\
\mathrm{L}_{70 \mathrm{~nm}}-40^{\circ} \mathrm{C}\end{array}$ & Yes & $* * * *$ & 4.40394E-06 & No & ns & $>0.999999999999$ \\
\hline $\begin{array}{c}\mathrm{L}_{30 \mathrm{~nm}}-40^{\circ} \mathrm{C} \text { vs. } \\
\mathrm{L}_{90 \mathrm{~nm}}-40^{\circ} \mathrm{C}\end{array}$ & Yes & $* * * *$ & $1.5316 \mathrm{E}-11$ & No & ns & $>0.999999999999$ \\
\hline $\begin{array}{c}\mathrm{L}_{30 \mathrm{~nm}}-40^{\circ} \mathrm{C} \text { vs. } \\
\mathrm{L}_{110 \mathrm{~nm}}-40^{\circ} \mathrm{C}\end{array}$ & No & ns & 0.752058186 & No & ns & 1 \\
\hline $\begin{array}{c}\mathrm{L}_{30 \mathrm{~nm}}-40^{\circ} \mathrm{C} \text { vs. } \\
\mathrm{L}_{110 \mathrm{~nm}}(\Delta 32)-40^{\circ} \mathrm{C}\end{array}$ & Yes & $* * * *$ & $<0.000000000000001$ & No & ns & $>0.999999999999$ \\
\hline $\begin{array}{c}\mathrm{L}_{30 \mathrm{~nm}}-55^{\circ} \mathrm{C} \text { vs. } \\
\mathrm{L}_{50 \mathrm{~nm}}-55^{\circ} \mathrm{C}\end{array}$ & Yes & $* * \star *$ & $<0.000000000000001$ & No & ns & 1 \\
\hline $\begin{array}{c}\mathrm{L}_{30 \mathrm{~nm}}-55^{\circ} \mathrm{C} \text { vs. } \\
\mathrm{L}_{70 \mathrm{~nm}}-55^{\circ} \mathrm{C}\end{array}$ & Yes & $* * * *$ & $<0.000000000000001$ & No & ns & 0.999915334 \\
\hline $\begin{array}{c}\mathrm{L}_{30 \mathrm{~nm}}-55^{\circ} \mathrm{C} \text { vs. } \\
\mathrm{L}_{90 \mathrm{~nm}}-55^{\circ} \mathrm{C}\end{array}$ & Yes & $* * * *$ & $<0.000000000000001$ & No & ns & 0.652751016 \\
\hline $\begin{array}{c}\mathrm{L}_{30 \mathrm{~nm}}-55^{\circ} \mathrm{C} \text { vs. } \\
\mathrm{L}_{110 \mathrm{~nm}}-55^{\circ} \mathrm{C}\end{array}$ & Yes & $* * * *$ & $<0.000000000000001$ & Yes & * & 0.046820994 \\
\hline $\begin{array}{c}\mathrm{L}_{30 \mathrm{~nm}}-55^{\circ} \mathrm{C} \text { vs. } \\
\mathrm{L}_{110 \mathrm{~nm}}(\Delta 32)-55^{\circ} \mathrm{C}\end{array}$ & Yes & $* * * *$ & $<0.000000000000001$ & No & ns & 0.99463928 \\
\hline
\end{tabular}




\begin{tabular}{|c|c|c|c|c|c|c|}
\hline $\begin{array}{c}\mathrm{L}_{50 \mathrm{~nm}}-40^{\circ} \mathrm{C} \text { vs. } \\
\mathrm{L}_{70 \mathrm{~nm}}-40^{\circ} \mathrm{C}\end{array}$ & Yes & $\star \star \star *$ & 0.000298267 & No & ns & $>0.999999999999$ \\
\hline $\begin{array}{l}\mathrm{L}_{50 \mathrm{~nm}}-40^{\circ} \mathrm{C} \text { vs. } \\
\mathrm{L}_{90 \mathrm{~nm}}-40^{\circ} \mathrm{C}\end{array}$ & No & ns & 0.337297518 & No & ns & >0.999999999999 \\
\hline $\begin{array}{c}\mathrm{L}_{50 \mathrm{~nm}}-40^{\circ} \mathrm{C} \text { vs. } \\
\mathrm{L}_{110 \mathrm{~nm}}-40^{\circ} \mathrm{C}\end{array}$ & Yes & $* * * *$ & $<0.000000000000001$ & No & ns & 0.999999987 \\
\hline $\begin{array}{c}\mathrm{L}_{50 \mathrm{~nm}}-40^{\circ} \mathrm{C} \text { vs. } \\
\mathrm{L}_{110 \mathrm{~nm}}(\Delta 32)-40^{\circ} \mathrm{C}\end{array}$ & Yes & $\star * \star \star *$ & $<0.000000000000001$ & No & ns & $>0.999999999999$ \\
\hline $\begin{array}{l}\mathrm{L}_{50 n m}-55^{\circ} \mathrm{C} \text { vs. } \\
\mathrm{L}_{70 n m}-55^{\circ} \mathrm{C}\end{array}$ & Yes & $* * * \star$ & $<0.000000000000001$ & No & ns & 0.999998522 \\
\hline $\begin{array}{c}\mathrm{L}_{50 \mathrm{~nm}}-55^{\circ} \mathrm{C} \text { vs. } \\
\mathrm{L}_{90 \mathrm{~nm}}-55^{\circ} \mathrm{C}\end{array}$ & Yes & $* \star \star \star$ & $<0.000000000000001$ & No & ns & 0.801620993 \\
\hline $\begin{array}{l}\mathrm{L}_{50 \mathrm{~nm}}-55^{\circ} \mathrm{C} \text { vs. } \\
\mathrm{L}_{110 \mathrm{~nm}}-55^{\circ} \mathrm{C}\end{array}$ & Yes & $\star * \star \star *$ & $<0.000000000000001$ & No & ns & 0.087276756 \\
\hline $\begin{array}{c}\mathrm{L}_{50 \mathrm{~nm}}-55^{\circ} \mathrm{C} \text { vs. } \\
\mathrm{L}_{110 \mathrm{~nm}}(\Delta 32)-55^{\circ} \mathrm{C}\end{array}$ & Yes & $* * * *$ & $<0.000000000000001$ & No & ns & 0.99939538 \\
\hline $\begin{array}{l}\mathrm{L}_{70 \mathrm{~nm}}-40^{\circ} \mathrm{C} \text { vs. } \\
\mathrm{L}_{90 \mathrm{~nm}}-40^{\circ} \mathrm{C}\end{array}$ & No & ns & 0.998819081 & No & ns & $>0.999999999999$ \\
\hline $\begin{array}{c}\mathrm{L}_{70 \mathrm{~nm}}-40^{\circ} \mathrm{C} \text { vs. } \\
\mathrm{L}_{110 \mathrm{~nm}}-40^{\circ} \mathrm{C}\end{array}$ & No & ns & 0.067160378 & No & ns & 0.999999999 \\
\hline $\begin{array}{c}\mathrm{L}_{70 \mathrm{~nm}}-40^{\circ} \mathrm{C} \text { vs. } \\
\mathrm{L}_{110 \mathrm{~nm}}(\Delta 32)-40^{\circ} \mathrm{C}\end{array}$ & Yes & $* \star \star \star$ & $<0.000000000000001$ & No & ns & $>0.999999999999$ \\
\hline $\begin{array}{c}\mathrm{L}_{70 \mathrm{~nm}}-55^{\circ} \mathrm{C} \text { vs. } \\
\mathrm{L}_{90 \mathrm{~nm}}-55^{\circ} \mathrm{C}\end{array}$ & Yes & 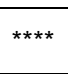 & $<0.000000000000001$ & No & ns & 0.983141406 \\
\hline $\begin{array}{c}\mathrm{L}_{70 \mathrm{~nm}}-55^{\circ} \mathrm{C} \text { vs. } \\
\mathrm{L}_{110 \mathrm{~nm}}-55^{\circ} \mathrm{C}\end{array}$ & Yes & $* * * *$ & $<0.000000000000001$ & No & ns & 0.304753716 \\
\hline $\begin{array}{c}\mathrm{L}_{70 \mathrm{~nm}}-55^{\circ} \mathrm{C} \text { vs. } \\
\mathrm{L}_{110 \mathrm{~nm}}(\Delta 32)-55^{\circ} \mathrm{C}\end{array}$ & Yes & $\star * \star \star *$ & $<0.000000000000001$ & No & ns & 0.999999984 \\
\hline $\begin{array}{c}\mathrm{L}_{90 \mathrm{~nm}}-40^{\circ} \mathrm{C} \text { vs. } \\
\mathrm{L}_{110 \mathrm{~nm}}-40^{\circ} \mathrm{C}\end{array}$ & Yes & $* * * \star$ & 7.48871E-06 & No & ns & 1 \\
\hline $\begin{array}{c}\mathrm{L}_{90 \mathrm{~nm}}-40^{\circ} \mathrm{C} \text { vs. } \\
\mathrm{L}_{110 \mathrm{~nm}}(\Delta 32)-40^{\circ} \mathrm{C}\end{array}$ & Yes & $* \star \star \star$ & $<0.000000000000001$ & No & ns & $>0.999999999999$ \\
\hline $\begin{array}{l}\mathrm{L}_{90 \mathrm{~nm}}-55^{\circ} \mathrm{C} \text { vs. } \\
\mathrm{L}_{110 \mathrm{~mm}}-55^{\circ} \mathrm{C}\end{array}$ & Yes & $\star * \star \star *$ & $<0.000000000000001$ & No & ns & 1 \\
\hline $\begin{array}{c}\mathrm{L}_{90 \mathrm{~nm}}-55^{\circ} \mathrm{C} \text { vs. } \\
\mathrm{L}_{110 \mathrm{~nm}}(\Delta 32)-55^{\circ} \mathrm{C} \\
\end{array}$ & Yes & $* * * *$ & $<0.000000000000001$ & No & ns & 0.999364715 \\
\hline $\begin{array}{c}\mathrm{L}_{110 \mathrm{~nm}}-40^{\circ} \mathrm{C} \text { vs. } \\
\mathrm{L}_{110 \mathrm{~nm}}(\Delta 32)-40^{\circ} \mathrm{C}\end{array}$ & Yes & $* * * *$ & $<0.000000000000001$ & No & ns & 0.99999998 \\
\hline $\begin{array}{c}\mathrm{L}_{110 \mathrm{~nm}}-55^{\circ} \mathrm{C} \text { vs. } \\
\mathrm{L}_{110 \mathrm{~nm}}(\Delta 32)-55^{\circ} \mathrm{C}\end{array}$ & Yes & $* * * *$ & $<0.000000000000001$ & No & ns & 0.561423678 \\
\hline
\end{tabular}




\section{Reference}

1. Ye, X.; Zheng, C.; Chen, J.; Gao, Y.; Murray, C. B., Using binary surfactant mixtures to simultaneously improve the dimensional tunability and monodispersity in the seeded growth of gold nanorods. Nano Lett. 2013, 13 (2), 765-71.

2. Orendorff, C. J.; Murphy, C. J., Quantitation of Metal Content in the Silver-Assisted Growth of Gold Nanorods. The Journal of Physical Chemistry B 2006, 110 (9), 3990-3994.

3. Bazrafshan, A.; Meyer, T. A.; Su, H.; Brockman, J. M.; Blanchard, A. T.; Piranej, S.;

Duan, Y.; Ke, Y.; Salaita, K., Tunable DNA Origami Motors Translocate Ballistically Over mum Distances at nm/s Speeds. Angew Chem Int Ed Engl 2020, 59 (24), 9514-9521.

4. Ke, Y.; Meyer, T.; Shih, W. M.; Bellot, G., Regulation at a distance of biomolecular interactions using a DNA origami nanoactuator. Nat. Commun. 2016, 7, 10935.

5. Petrosyan, R., Improved approximations for some polymer extension models. Rheol. Acta 2017, 56 (1), 21-26.

6. Zhang, Y.; Qiu, Y.; Blanchard, A. T.; Chang, Y.; Brockman, J. M.; Ma, V. P.; Lam, W. A.; Salaita, K., Platelet integrins exhibit anisotropic mechanosensing and harness piconewton forces to mediate platelet aggregation. Proc. Natl. Acad. Sci. U.S.A 2018, 115 (2), 325-330.

7. Liedl, T.; Högberg, B.; Tytell, J.; Ingber, D. E.; Shih, W. M., Self-assembly of threedimensional prestressed tensegrity structures from DNA. Nat. Nanotechnol. 2010, 5, 520.

8. Castro, C. E.; Su, H.-J.; Marras, A. E.; Zhou, L.; Johnson, J., Mechanical design of DNA nanostructures. Nanoscale 2015, 7 (14), 5913-5921.

9. Woodside, M. T.; Behnke-Parks, W. M.; Larizadeh, K.; Travers, K.; Herschlag, D.; Block, S. M., Nanomechanical measurements of the sequence-dependent folding landscapes of single nucleic acid hairpins. Proc. Natl. Acad. Sci. U.S.A 2006, 103 (16), 6190-5. 10. Bell, G. I., Models for the specific adhesion of cells to cells. Science 1978, 200 (4342), 618-27.

11. Ouldridge, T. E.; Louis, A. A.; Doye, J. P. K., Structural, mechanical, and thermodynamic properties of a coarse-grained DNA model. The Journal of chemical physics 2011, 134 (8), 085101.

12. Šulc, P.; Romano, F.; Ouldridge, T. E.; Rovigatti, L.; Doye, J. P. K.; Louis, A. A., Sequence-dependent thermodynamics of a coarse-grained DNA model. The Journal of chemical physics 2012, 137 (13), 135101.

13. Snodin, B. E. K.; Randisi, F.; Mosayebi, M.; Šulc, P.; Schreck, J. S.; Romano, F.; Ouldridge, T. E.; Tsukanov, R.; Nir, E.; Louis, A. A.; Doye, J. P. K., Introducing improved structural properties and salt dependence into a coarse-grained model of DNA. The Journal of chemical physics 2015, 142 (23), 234901.

14. Snodin, B. E. K.; Schreck, J. S.; Romano, F.; Louis, A. A.; Doye, J. P. K., Coarse-grained modelling of the structural properties of DNA origami. Nucleic acids research 2019, 47 (3), 1585-1597.

15. Bai, X.-c.; Martin, T. G.; Scheres, S. H. W.; Dietz, H., Cryo-EM structure of a 3D DNAorigami object. Proc. Natl. Acad. Sci. U.S.A 2012, 109 (49), 20012-20017.

16. Chhabra, H.; Mishra, G.; Cao, Y.; Prešern, D.; Skoruppa, E.; Tortora, M. M. C.; Doye, J. P. K., Computing the Elastic Mechanical Properties of Rodlike DNA Nanostructures. Journal of chemical theory and computation 2020, 16 (12), 7748-7763. 
17. Engel, M. C.; Smith, D. M.; Jobst, M. A.; Sajfutdinow, M.; Liedl, T.; Romano, F.; Rovigatti, L.; Louis, A. A.; Doye, J. P. K., Force-Induced Unravelling of DNA Origami. ACS Nano 2018, 12 (7), 6734-6747.

18. Romano, F.; Chakraborty, D.; Doye, J. P. K.; Ouldridge, T. E.; Louis, A. A., Coarsegrained simulations of DNA overstretching. The Journal of chemical physics 2013, 138 (8), 085101.

19. Mosayebi, M.; Romano, F.; Ouldridge, T. E.; Louis, A. A.; Doye, J. P. K., The Role of Loop Stacking in the Dynamics of DNA Hairpin Formation. The Journal of Physical Chemistry $B$ 2014, 118 (49), 14326-14335.

20. Engel, M. C.; Romano, F.; Louis, A. A.; Doye, J. P. K., Measuring Internal Forces in Single-Stranded DNA: Application to a DNA Force Clamp. Journal of chemical theory and computation 2020, 16 (12), 7764-7775.

21. Nickels, P. C.; Wunsch, B.; Holzmeister, P.; Bae, W.; Kneer, L. M.; Grohmann, D.; Tinnefeld, P.; Liedl, T., Molecular force spectroscopy with a DNA origami-based nanoscopic force clamp. Science 2016, 354 (6310), 305-307.

22. Rovigatti, L.; Šulc, P.; Reguly, I. Z.; Romano, F., A comparison between parallelization approaches in molecular dynamics simulations on GPUs. Journal of Computational Chemistry 2015, $36(1), 1-8$.

23. Russo, J.; Tartaglia, P.; Sciortino, F., Reversible gels of patchy particles: Role of the valence. The Journal of chemical physics 2009, 131 (1), 014504.

24. Kube, M.; Kohler, F.; Feigl, E.; Nagel-Yüksel, B.; Willner, E. M.; Funke, J. J.; Gerling, T.; Stömmer, P.; Honemann, M. N.; Martin, T. G.; Scheres, S. H. W.; Dietz, H., Revealing the structures of megadalton-scale DNA complexes with nucleotide resolution. Nature

Communications 2020, 11 (1), 6229.

25. Cooper, M.; Ebner, A.; Briggs, M.; Burrows, M.; Gardner, N.; Richardson, R.; West, R., Cy3B: improving the performance of cyanine dyes. J Fluoresc 2004, 14 (2), 145-50.

26. Sato Matsuo, E.; Tanaka, T., Kinetics of discontinuous volume-phase transition of gels.

The Journal of chemical physics 1988, 89 (3), 1695-1703.

27. Wang, J.; Gan, D.; Lyon, L. A.; El-Sayed, M. A., Temperature-jump investigations of the kinetics of hydrogel nanoparticle volume phase transitions. J. Am. Chem. Soc 2001, 123 (45), 11284-9.

28. Reese, C. E.; Mikhonin, A. V.; Kamenjicki, M.; Tikhonov, A.; Asher, S. A., Nanogel nanosecond photonic crystal optical switching. J. Am. Chem. Soc 2004, 126 (5), 1493-6.

29. Ahmed, Z.; Gooding, E. A.; Pimenov, K. V.; Wang, L.; Asher, S. A., UV resonance Raman determination of molecular mechanism of poly(N-isopropylacrylamide) volume phase transition. J Phys Chem B 2009, 113 (13), 4248-56.

30. Wu, T. Y.; Zrimsek, A. B.; Bykov, S. V.; Jakubek, R. S.; Asher, S. A., Hydrophobic Collapse Initiates the Poly ( N-isopropylacrylamide) Volume Phase Transition Reaction Coordinate. J Phys Chem B 2018, 122 (11), 3008-3014.

31. Wahrmund, J.; Kim, J.-W.; Chu, L.-Y.; Wang, C.; Li, Y.; Fernandez-Nieves, A.; Weitz, D. A.; Krokhin, A.; Hu, Z., Swelling Kinetics of a Microgel Shell. Macromolecules 2009, 42 (23), 9357-9365.

32. Suarez, I. J.; Fernandez-Nieves, A.; Marquez, M., Swelling kinetics of poly(Nisopropylacrylamide) minigels. J Phys Chem B 2006, 110 (51), 25729-33. 
33. Zhao, J.; Su, H.; Vansuch, G. E.; Liu, Z.; Salaita, K.; Dyer, R. B., Localized Nanoscale Heating Leads to Ultrafast Hydrogel Volume-Phase Transition. ACS Nano 2019, 13 (1), 515 525. 